\title{
Electrical and Magnetic Investigations of Magnesium-doped Epitaxial Gadolinium Nitride Thin Films
}

by

\section{Chang-Min Lee}

A thesis submitted to the Victoria University of Wellington in fulfilment of the requirements for the degree of Master of Science in Physics

Victoria University of Wellington 2015 


\begin{abstract}
Mg-doped epitaxial GdN thin films with various Mg-doping levels were grown using molecular beam epitaxy, and their electric, magnetic and optoelectronic properties were investigated. Characterisation through X-ray diffraction technique showed that there is no systematic variation in the crystallographic structure of the films with increasing level of $\mathrm{Mg}$-doping, for $\mathrm{Mg}$ concentrations up to $\sim 5 \times 10^{19}$ atoms $/ \mathrm{cm}^{3}$. However, from $\mathrm{Mg}$ concentration $\sim 2 \times 10^{20}$ atoms $/ \mathrm{cm}^{3}$ a clear deterioration in the crystalline quality was seen. We observed an increase in the resistivity of the films from $0.002 \Omega \mathrm{cm}$ to $600 \Omega \mathrm{cm}$ at room temperature when increasing the $\mathrm{Mg}$ doping level, resulting in semi-insulating films for $\mathrm{Mg}$ concentrations up to $5 \times 10^{19}$ atoms $/ \mathrm{cm}^{3}$. Hall effect measurements revealed that the n-type carrier concentration was reduced from $7 \times 10^{20} \mathrm{~cm}^{-3}$ for an undoped film to $5 \times 10^{15} \mathrm{~cm}^{-3}$ for a heavily doped film, demonstrating electron compensation in GdN via Mg-doping. Magnetic measurements exhibited substantial contrasts in the films, with a Curie temperature of $\sim 70 \mathrm{~K}$ for an undoped film reduced down to $\sim 50 \mathrm{~K}$ for a heavily Mg-doped film. Finally, photoconductivity measurements showed that films with higher level of Mg-doping displaying a faster photoconductive response. The decay time of $13000 \mathrm{~s}$ for an undoped film was reduced to $170 \mathrm{~s}$ with a moderate level of Mg-doping, which raises the possibility of $\mathrm{Mg}$ impurities providing hole traps that act as recombination centres in n-type GdN films.
\end{abstract}




\section{Constants used in this thesis}

\begin{tabular}{lll}
\hline Symbol & Quantity & Value \\
\hline$\mu_{0}$ & Permeability of free space & $4 \pi \times 10^{-7} \mathrm{Vs} / \mathrm{Am}$ \\
$\mu_{B}$ & Bohr magneton & $9.27400968(20) \times 10^{-24} \mathrm{JT}^{-1}$ \\
$k_{B}$ & Boltzmann constant & $1.3806488(13) \times 10^{-23} \mathrm{JK}^{-1}$ \\
$\hbar$ & Reduced Planck constant & $1.054571726(47) \times 10^{-34} \mathrm{Js}$ \\
$e$ & Elementary charge & $1.602176565(35) \times 10^{-19} \mathrm{C}$ \\
\hline
\end{tabular}




\section{Contents}

1 Introduction 2

1.1 Rare-earth Nitride . . . . . . . . . . . . . . . . . 2

1.2 Background and Initial Results . . . . . . . . . . . . . . 4

1.3 Aim . . . . . . . . . . . . . . 6

2 Growth and Structural Properties $\quad 8$

2.1 Sample Growth . . . . . . . . . . . . . . . 8

2.2 Structural Properties . . . . . . . . . . . . . . . 13

3 Electrical and Magnetic Properties 20

3.1 Electrical Characterisation Techniques . . . . . . . . . 20

3.2 Electrical Properties . . . . . . . . . . . . . . 22

3.3 Magnetic Characterisation Techniques . . . . . . . . . 27

3.4 Magnetic Properties . . . . . . . . . . . . . . . . . 29

3.5 Polaron Scenario . . . . . . . . . . . . . . . . . . . 34

4 Photoconductivity $\quad 36$

4.1 Photoconductivity Characterisation Techniques . . . . . . 36

4.2 Temperature-dependent Photoconductivity . . . . . . . . . . 38

4.3 Persistent Photoconductivity . . . . . . . . . . . . . 43

5 Conclusion 48 


\section{Chapter 1}

\section{Introduction}

\section{$1.1 \quad$ Rare-earth Nitride}

The rare-earths (RE) are elements with atomic number ranging from 57 (lanthanum) to 71 (lutetium), comprising elements whose $4 f$ orbitals are filled, with some of the series (lanthanum, cerium, gadolinium, lutetium) having an additional $5 d$ electron. Being the only stable elements with more than marginally filled $f$-shell (with the exception of promethium which has no stable isotope), they have the largest spin and orbital moments, ensuring their utility in technologies that require strong permanent magnets, such as wind turbines and batteries for electrical cars. ${ }^{1}$

The term "rare-earth" can be misleading as these metals are not particularly rare or precious. There are found with abundance in the earth's crust at concentration exceeding $0.5 \mathrm{ppm}$ which is more abundant than metals like silver and mercury, as we can see in Figure $(1.1) .^{2}$ For the past decades there has been enough advances in the techniques and technology to separate the members of the RE, and RE pellets with purity of up $99.999 \%$ are commercially available. Typically the purity of the RE source is about $99.99 \%$ by weight with the main impurities being oxygen, carbon, nitrogen, calcium and iron.

$\mathrm{RE}$ elements can combine with nitrogen to form rare-earth mononitride (REN) compounds, all with the $\mathrm{FCC} \mathrm{NaCl}$ crystal structure and lattice constants varying from $\sim 0.48 \mathrm{~nm}(\mathrm{LuN})$ to $\sim 0.53 \mathrm{~nm}(\mathrm{LaN})$ across the series. ${ }^{1}$ From a historical point of view the RENs were explored extensively in the 1960s, and 1970s,${ }^{3}$ but due to their propensity for nitrogen vacancy 


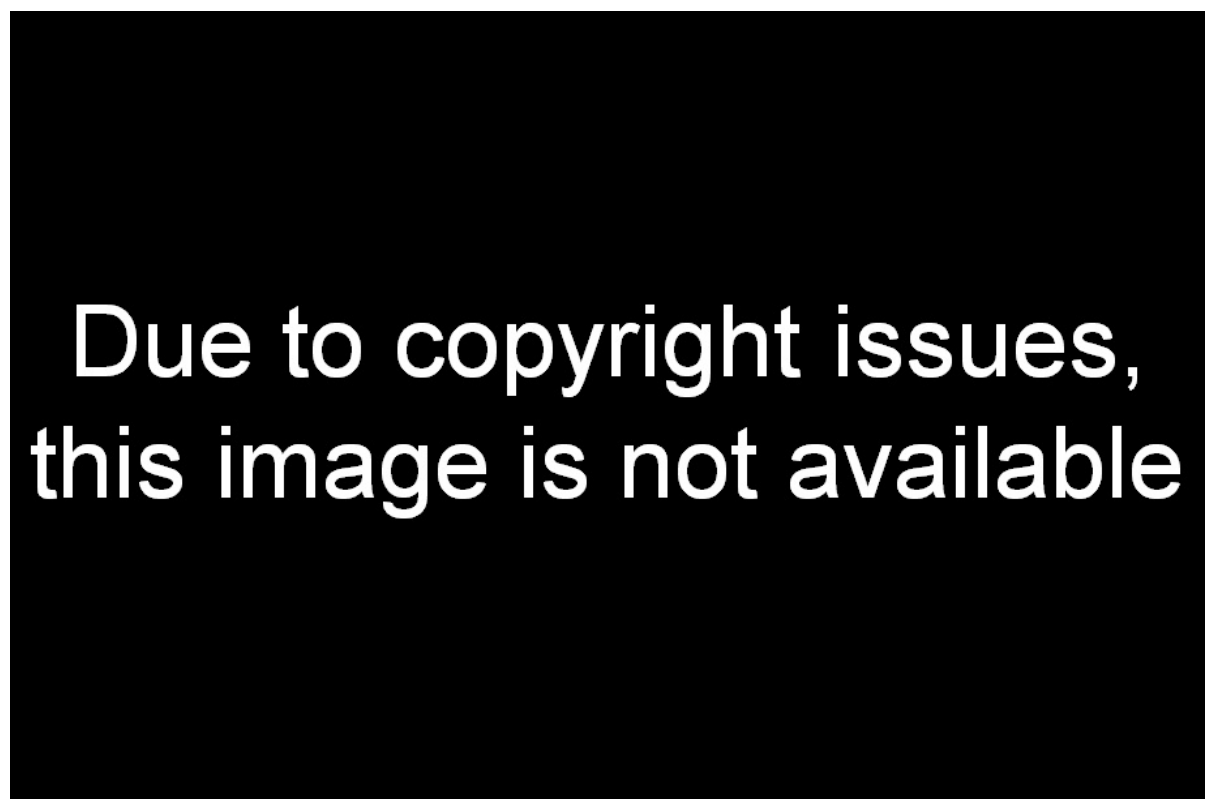

Figure 1.1: The relative abundance of elements in Earth's upper continental crust, with the rare-earth elements highlighted in red.

$\left(V_{N}\right)$, and when exposed to air, for oxide formation, their fundamental properties, such as the electrical conductivity or magnetic state, were far from clear. ${ }^{4}$ These impediments have finally been overcome by advances in thin film fabrication with ultra-high vacuum based growth technology such as molecular beam epitaxy (MBE), pulsed-laser deposition (PLD), and DC/RF magnetron sputtering. They are the techniques of choice for deposition of RE-based materials because the high-vacuum and inert-gas environment helps to ensure material purity and interface quality and these methods are subsequently the most common used in this field. RENs have been grown recently with sufficient stoichiometry to establish their properties confidently, and effective capping layer, to prevent decomposition of REN films in air, have also been developed. Most of the capping layers used are polycrystalline AlN and GaN thin films, as these layers are transparent and relatively easy to grow.

Currently, the field of research into the RENs is growing rapidly, mainly because both semiconducting and ferromagnetic properties have been established in most of the 14 REN compounds. ${ }^{1}$ Thus RENs contribute new members to intrinsic ferromagnetic semiconductors, a very rare class of materials. One of the reasons for the interest in REN lies on the strong coupling between the spin and electric charge degrees of freedom due to the overlap of localised rare-earth $4 f$ electrons and the delocalised nitrogen $2 p$, and the 
RE $6 s$ and $5 d$ electrons. Such coupling is likely to have a significant contribution to the intrinsic ferromagnetic semiconductor nature of the RENs. ${ }^{5}$ This implies that their doping level can potentially be controlled without affecting their magnetic properties, leading to more possibilities in the application and fundamental research of spintronics. Thus intrinsic ferromagnetic semiconductors have advantages over dilute magnetic semiconductors (DMS). DMSs rely on the presence of foreign magnetic ions such as $\mathrm{Mn}$ or Gd for their magnetic properties, not allowing the independent control of their doping level and magnetic properties. ${ }^{6}$ Also, incorporating a high concentration of foreign ions in DMS is often compromised by its solubility limit, thus the growth of high quality DMS is difficult. ${ }^{7}$

In addition, the strong coupling between the charge and spin in these RENs means that only the majority spin bands are occupied by the electrons and holes, leaving the minority-spin band edges unoccupied. ${ }^{1}$ This provides a potential for REN to be exploited for any device that requires transport of carriers with only majority-spin state such as spin light-emitting diode and spin filter. ${ }^{8}$

It is worth pointing out that the spintronics group at Victoria University of Wellington have demonstrated that RENs have magnetic properties that provide interesting contrasts and promising complementary electronic properties. For example, GdN films have a huge magnetic moment of $7 \mu_{B}$ per $\mathrm{Gd}$ ion and a coercive field as small as $0.01 \mathrm{~T},{ }^{9,10,11,12}$ while in contrast $\mathrm{SmN}$ has a magnetic moment of only $0.035 \mu_{B}$ per Sm ion and a coercive field in excess of $6 \mathrm{~T} .{ }^{13}$ The three-decade contrast in coercive field makes these two RENs ideal for a hard- and soft-ferromagnetic pair, for non-volatile magnetoresistance memory (MRAM application). ${ }^{1}$

Overall, the interest for the study of RENs is driven by their intrinsic ferromagnetic semiconducting nature, which is a very rare property. Growth techniques have been developed for REN to ensure good stoichiometry and provide good passivation against oxidation in air. They offer a lot of opportunities with potential applications in semiconductor-based spintronics.

\subsection{Background and Initial Results}

The most thoroughly studied REN is gadolinium nitride $(\mathrm{GdN})$, in part because of the maximum spin moment of $7 \mu_{B}$ and zero orbital angular momentum in the half-filled $4 f$ shell of $\mathrm{Gd}^{3+}$. Thus the magnetic moment of 
Gd has spin contribution only, which is a unique feature among the REs. GdN has also the highest Curie temperature $\left(T_{C}\right)$ of the series, reported as $70 \mathrm{~K}$ in most studies of the past 50 years. ${ }^{11,14,15,16,17}$ However a recent report suggests that $T_{C}$ has been enhanced by magnetic polarons from less than $50 \mathrm{~K}$ in undoped epitaxial GdN films. ${ }^{18}$ This matter will be discussed further in Chapter 3. Historically, the conductive nature of GdN was uncertain, due to the unintentional n-type doping by high concentration of $V_{N}$. Polycrystalline GdN films grown at room temperature present low $V_{N}$ densities, with resistivity comparable to moderately doped semiconductors. It has been shown that the resistivity of GdN can be tuned by carefully controlling the growth parameters, such as the $\mathrm{N}_{2}$ partial pressure. ${ }^{19}$ Interestingly, this paper shows a strong interplay between charge carriers and magnetism but was not able to conclude about the mechanism for the interplay.

The improvement of the crystallographic properties of GdN films, with the recent achievement of epitaxially grown high quality films have now provided a better understanding and picture of both the electrical and magnetic properties. In addition, this provides an access to well-ordered films which is mandatory for the fabrication of REN-based electronic/spintronics devices.

Nominally undoped epitaxial GdN thin films typically show an n-type conductivity, primarily due to doping by $V_{N} \cdot{ }^{3}$ A literature study shows that there is a wide range of epitaxial GdN thin films reported with electron carrier densities typically above $10^{20} \mathrm{~cm}^{-3}$ at room temperature..$^{7,9,17,20}$ So far, despite the advances in thin film growth, it has remained difficult to reduce the concentration of $V_{N}$ much below the $1 \%$ level, which dopes the films with as many as three electrons per $V_{N}$. Such relatively high $V_{N}$ concentrations will remain a problem, in view of the epitaxial growth temperature, typically above $500{ }^{\circ} \mathrm{C}$, and the small $V_{N}$ formation energy of $\sim 0.5 \mathrm{eV} .^{21}$ From a device perspective it will be crucial to decrease the conductivity. Semi-insulating and insulating GdN layers could be useful, in combination with group-III nitrides, for fabrication of spintronic, electronic and optoelectronic devices. Incorporation of such layer may avoid leakage current or degradation of radio frequency performance of the devices.

The spintronics group at Victoria University of Wellington has already developed a "savoir-faire" to grow high quality thin films, being to date the only group growing epitaxial films of any besides GdN, including SmN and $\mathrm{EuN}$, a facility required for the development of electronic and spintronic devices. $^{22}$ This is done through the use of a molecular beam epitaxy (MBE) system. Such epitaxial GdN, SmN and EuN thin films with high crystalline 
quality are suitable for the investigation of their electric and magnetic properties. ${ }^{17,23,24,25}$

Most of the grown epitaxial thin films of GdN were found to have a high $T_{C}$ of $\sim 70 \mathrm{~K}$ in agreement with the reported value in the literature. ${ }^{11,14,15,16,17}$ They had n-type carrier concentration ranging from $10^{20}$ to $10^{22} \mathrm{~cm}^{-3}$ and resistivity in the order of 0.05 to $10 \mathrm{~m} \Omega . c m$ at room temperature, ${ }^{26}$ which suggested high levels of $V_{N}$ in the grown GdN films, as each $V_{N}$ is able to provide up to 3 electrons, effectively acting as an electron donor. ${ }^{21}$ In contrast, polycrystalline thin films of GdN are typically observed to have relatively high resistivity in the order of 0.1 to $10 \Omega . c m$ at room temperature, about three orders of magnitude greater than that of epitaxial films. ${ }^{12,16,19,27}$ This is because temperature of several hundred ${ }^{\circ} \mathrm{C}$ is required for epitaxial film growth, whereas polycrystalline film growth is done at room temperature. Since the formation energy of $V_{N}$ is relatively low, the $V_{N}$ concentration is higher for films grown at higher temperature (typically $\sim 1 \%$ for epitaxial films), resulting in more carriers and thus lower resistivity. ${ }^{28}$ It was also observed that increasing the nitrogen pressure by a factor of 10 results in increasing the resistivity of GdN film by a factor of $10 .{ }^{19}$

In 2013, my supervisor, Dr Franck Natali, and his collaborators at the CRHEA-CNRS-Valbonne, France, started a preliminary study of electron compensation in GdN via Mg-doping. The first attempt looked promising, and a provisional patent has been launched. The resistivity and Hall effect measurements showed that the undoped GdN has a resistivity of $\sim 2 \times 10^{-3}$ $\Omega \mathrm{cm}$ and a carrier concentration of $\sim 6.5 \times 10^{20} \mathrm{~cm}^{-3}$. Mg-doped GdN films with $\mathrm{Mg}$ concentration of $\sim 1 \times 10^{19} \mathrm{~cm}^{-3}$ and $\sim 5 \times 10^{19} \mathrm{~cm}^{-3}$ (measured by secondary ion mass spectrometry) had resistivity of $\sim 25 \Omega \mathrm{cm}$ and $>10^{4}$ $\Omega \mathrm{cm}$ respectively, which is much higher than the undoped GdN. This clearly suggests a way to control the carrier concentration of the GdN thin film.

\subsection{Aim}

My Master's thesis work focused on the epitaxial growth of GdN thin films while reducing the unintentional n-type residual doping caused by $V_{N}$. To achieve this, we explored the compensation of the electron doping in GdN films from the $V_{N}$ by the introduction of $\mathrm{Mg}$ atom. $\mathrm{Mg}^{2+}$ is expected to act as an acceptor in $\mathrm{GdN}$ since it donates 1 less electron than $\mathrm{Gd}^{3+} .29$ 
In Chapter 2, the growth procedures and the structural properties of $\mathrm{Mg}$ doped epitaxial GdN thin films are outlined and discussed. I carried out the growth of the films in France alongside our long-term French collaborates from CNRS-CRHEA at Valbonne, who are involved in the Marsden project (see below). An MBE system was used for the growth, using conventional effusion cell to evaporate $\mathrm{Gd}$ and $\mathrm{Mg}$ in the presence of $\mathrm{NH}_{3}$. X-ray diffraction (XRD) technique was also used at CNRS-CRHEA to investigate the structural properties of the films. This allowed the investigation of how the crystal structure changes with higher Mg-doping level and how much $\mathrm{Mg}$ incorporation causes a significant deterioration in the crystalline quality.

In Chapter 3, the electrical and magnetic properties of Mg-doped epitaxial GdN thin film are outlined and discussed. For electrical characterisation, Hall effect and resistivity measurements were carried out at room temperature using the van der Pauw geometry to see if the carrier concentration is actually reduced by the Mg-doping. This was followed by temperaturedependent resistivity measurements, which was carried out using a liquid He-cooled cryostat. For magnetic characterisation, field-dependent and temperature-dependent magnetisation measurements were carried out using a superconducting quantum interference device (SQUID). The temperaturedependent resistivity and magnetisation measurements show interesting interplay between charge carriers and magnetism, and this matter is discussed in the "Polaron Scenario" section.

In Chapter 4, the photoconductive properties of Mg-doped epitaxial GdN thin film are outlined and discussed. This was carried out to investigate the effect of photo-excitation on the carrier concentration. Temperaturedependent resistivity measurements were carried out in the presence of HeNe laser, to see if the samples exhibited photoconductivity. Furthermore, to probe the effect of $\mathrm{Mg}$-doping on the impurity levels in GdN, time-dependent measurements of photoconductivity were carried out and persistent photoconductivity (PPC) was observed.

My Master's thesis work has been part of the Marsden project "Semiconductorbased spintronics: Can rare-earth nitrides and group III-nitrides get it together". In January 2015, my work on the electrical and magnetic properties of Mg-doped epitaxial GdN thin films was published in Applied Physics Letters (Lee et al.). ${ }^{30}$ The paper demonstrates the effective electron compensation in epitaxial GdN by Mg-doping, which was the main focus of my work. A large part of my thesis is based on this paper. 


\section{Chapter 2}

\section{Growth and Structural Properties}

\subsection{Sample Growth}

In this section, the experimental procedures taken to prepare the epitaxial GdN thin film samples are outlined. I visited CNRS-CRHEA, Valbonne, France and used the Riber molecular beam epitaxy (MBE) system for the growth of Mg-doped epitaxial GdN thin films. A schematic digram of an MBE system is shown in Figure (2.1).

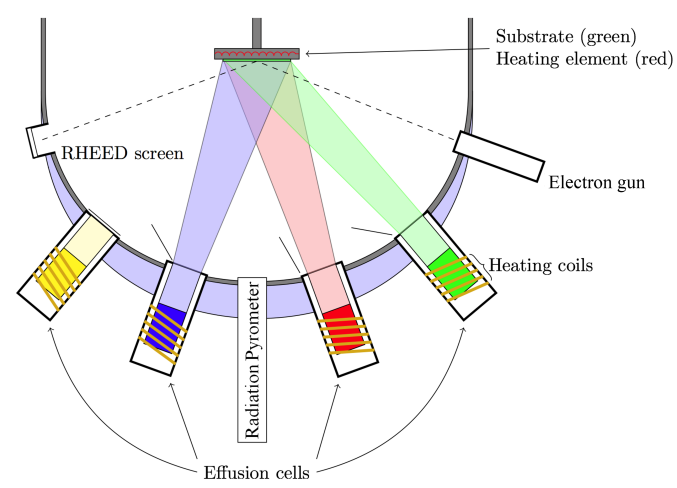

Figure 2.1: A schematic diagram of an MBE system. It is equipped with conventional effusion cells for $\mathrm{Al}, \mathrm{Ga}, \mathrm{Mg}$ and Gd solid sources. The substrate holder can be heated for higher substrate temperature during the growth. The electron gun allows in situ surface analysis during the growth. The temperature is measured using the pyrometer. 
MBE has been the technique of choice, because the high vacuum and inertgas environment ensure that good crystal quality and material purity are achieved for the growth of RE-based materials. ${ }^{1}$ Indeed, it has been demonstrated that growth by MBE tends to be the best growth method to achieve rapid improvement in the quality of GdN films. ${ }^{7,9,22}$
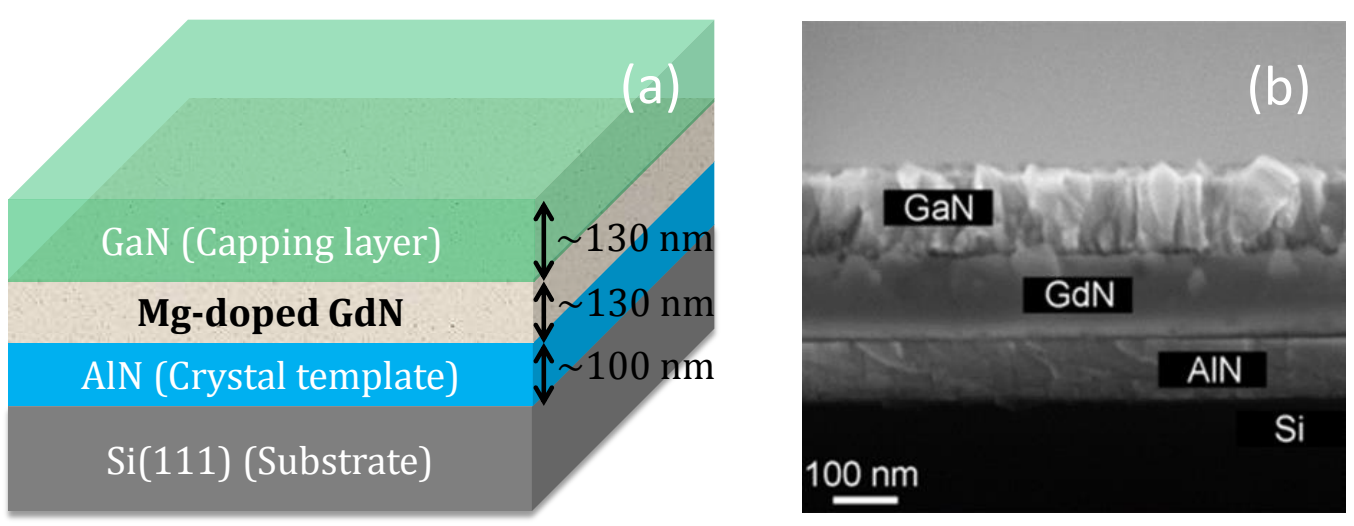

Figure 2.2: (a) a diagram for the film structure of the grown samples and (b) an SEM image of an undoped epitaxial GdN thin film.

The film structure of the grown samples can be seen in Figure (2.2a) and the SEM image in Figure (2.2b). The Mg-doped GdN layers were grown by MBE on $100 \mathrm{~nm}$ thick [0001]-orientated wurtzite aluminium nitride (AlN) buffer layer, which were themselves grown by MBE on [111]-orientated 2inch silicon wafer. The sample was cut into square pieces of size $1 \mathrm{~cm} \times$ $1 \mathrm{~cm}$, and GdN was grown on top of the AlN buffer layer with different growth condition for each $1 \mathrm{~cm} \times 1 \mathrm{~cm}$ piece.

The GdN layer is grown on top of the AlN buffer layer for mainly two reasons. First, Gd tends to easily react with $\mathrm{Si}$ to form silicides, which is undesirable for GdN growth. This is prevented by having an AlN layer between Gd and Si. Also, it has been demonstrated that the $\operatorname{AlN}(0001)$ layer encourages the epitaxial growth of GdN. ${ }^{7}$ The GaN is grown on top of the GdN layer to prevent decomposition of $\mathrm{GdN}$ in air. GaN is a suitable choice for capping layer because its transparency makes it more adequate for optical measurements, and its high electrical resistivity means that it will have little interference with the grown GdN when electrical measurements are carried out. Ammonia $\left(\mathrm{NH}_{3}\right)$ was used as the nitrogen precursor, which produced atomic nitrogen species $(\mathrm{N})$ by thermally activated decomposition on the growing surface. Conventional effusion cells were used for Al, Ga, $\mathrm{Mg}$ and Gd solid sources. 
Growth Procedure:

1. The AlN/Si sample is introduced into a buffer chamber and loaded on a substrate outgassing stage, to do a $300{ }^{\circ} \mathrm{C}$ bake-out for outgassing water vapour from the substrate and the sample holder.

2. The sample is then introduced into the growth chamber, and the AlN surface is cleaned by thermal annealing at $880{ }^{\circ} \mathrm{C}$ under $\mathrm{NH}_{3}$ prior to the growth. Such thermal annealing is well-known to optimise the nucleation layer and subsequent growth steps.

3. GdN is grown by evaporating Gd pellets from a high temperature effusion cell in the presence of $\mathrm{NH}_{3}$. The beam equivalent pressure (BEP) of $\mathrm{Gd}$ and $\mathrm{NH}_{3}$ were $5 \times 10^{-8}$ Torr and $1.9 \times 10^{-5}$ Torr, respectively. The substrate temperature is maintained at $650{ }^{\circ} \mathrm{C}$ because high temperature is required for epitaxial growth. The BEP of $\mathrm{Mg}$ ranged from $5 \times 10^{-11}$ Torr to $6.5 \times 10^{-8}$ Torr corresponding to $\mathrm{Mg}$ effusion cell temperature between 162 and $300{ }^{\circ} \mathrm{C}$. The growth rate of the GdN:Mg layer was $\sim 0.15 \mu \mathrm{m} / \mathrm{h}$.

4. Lastly, GaN layer is grown on top of the GdN layer by evaporating $\mathrm{Ga}$ atoms in the presence of $\mathrm{NH}_{3}$.

The temperature of the substrate was monitored with a disappearing-filament pyrometer. The observation by in situ reflection high-energy electron diffraction (RHEED) of the well-known $(7 \times 7 \longleftrightarrow 1 \times 1)$ Si(111) surface phase transition was used to calibrate the pyrometer to get a transition temperature of $830{ }^{\circ} \mathrm{C}$. The effusion cells were outgassed before introducing the substrate into the growth chamber for better purity.

As shown in Figure (2.3), only the Mg effusion cell temperature is varied and otherwise the same growth conditions are used for the all Mg-doped GdN films. Bayard-Alpert ionization gauge is used to monitor the pressure of the chamber. The pressure is noted for both when the $\mathrm{Mg}$ effusion cell shutter is open and closed, and taking the difference in pressure gives the $\mathrm{Mg}$ BEP. For $\mathrm{Mg}$ effusion cell temperature ranging $160 \sim 300{ }^{\circ} \mathrm{C}$, the $\mathrm{Mg}$ $\mathrm{BEP}$ increases exponentially with increasing $\mathrm{Mg}$ effusion cell temperature, as observed in Figure (2.4). 


\begin{tabular}{|c|c|c|c|c|}
\hline \multirow[t]{2}{*}{ Sample } & \multirow{2}{*}{$\begin{array}{l}\text { Growth } \\
\text { Temp. }\left({ }^{\circ} \mathrm{C}\right)\end{array}$} & $\begin{array}{l}\text { Effusion } \\
\text { Cell Temp. }\end{array}$ & $\begin{array}{l}\text { Effusion } \\
\text { Cell BEP }\end{array}$ & \multirow[t]{2}{*}{ Thickness (nm) } \\
\hline & & $\mathrm{Mg}\left({ }^{\circ} \mathrm{C}\right)$ & Mg (Torr) & \\
\hline SC0910 & 650 & - & - & 140 \\
\hline Sc0917 & 650 & 200 & $1.3 \times 10^{-9}$ & 140 \\
\hline Sc0919 & 650 & 253 & $8.0 \times 10^{-9}$ & 140 \\
\hline sc0981 & 650 & 162 & $1.28 \times 10^{-10}$ & 130 \\
\hline SCO982 & 650 & 300 & $6.49 \times 10^{-8}$ & 130 \\
\hline Sc0983 & 650 & 180 & $2.09 \times 10^{-10}$ & 130 \\
\hline Sc0984 & 650 & 253 & $8.16 \times 10^{-9}$ & 130 \\
\hline SC0985 & 650 & 220 & $2.17 \times 10^{-9}$ & 130 \\
\hline SC0987 & 650 & - & - & 130 \\
\hline SC0988 & 650 & 200 & $5.94 \times 10^{-10}$ & 130 \\
\hline
\end{tabular}

Figure 2.3: Different growth conditions for the grown Mg-doped GdN thin films are shown. Note that the samples SCO981 SCO988 were grown by myself, whereas samples SCO910 SCO919 were grown by Franck Natali in 2013.

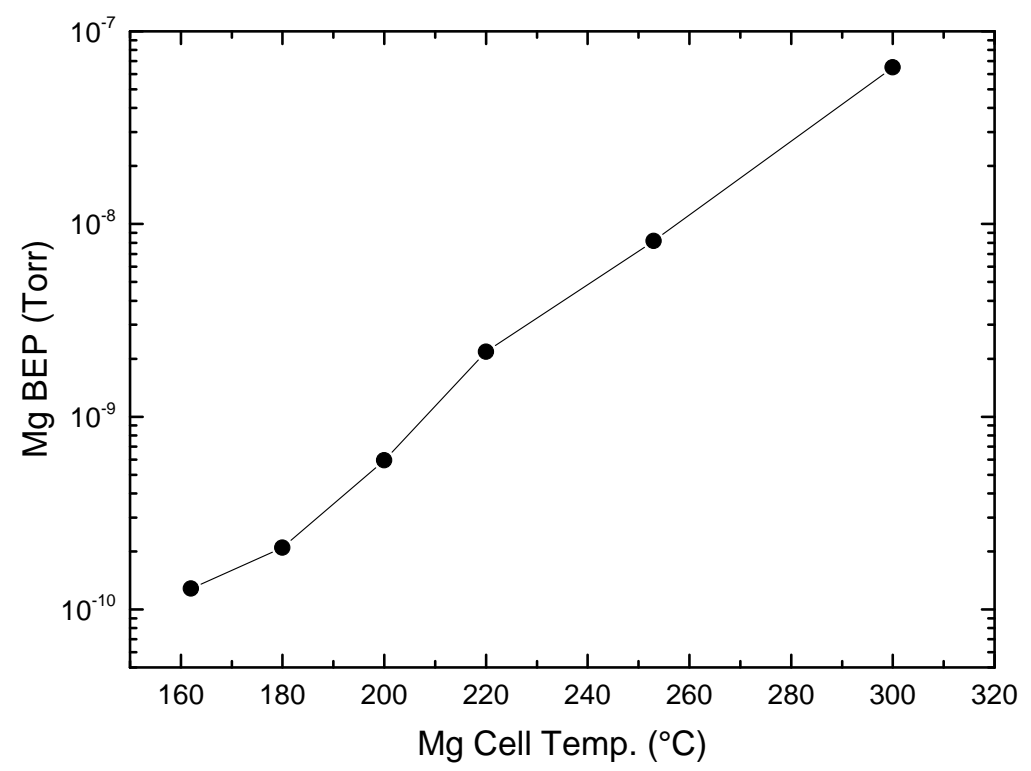

Figure 2.4: $\mathrm{Mg} \mathrm{BEP}$ as a function of $\mathrm{Mg}$ effusion cell temperature for samples SCO981 SCO988. 
Zehe et al. gives a simple explanation for the observed exponential behaviour using kinetic gas theory. ${ }^{31}$ The particle flux is given by $j=n \tilde{v} / 4$ where $n$ is the particle density and $\tilde{v}$ is the average particle speed. Since $n=p / k_{B} T$ and $\tilde{v}=\sqrt{8 k_{B} T / \pi m}$ in simple kinetic theory of gas, combining these lead to

$$
j=\frac{p}{\sqrt{2 \pi m k_{B} T}}
$$

where $p$ is the partial pressure given by $p=p_{0} \exp \left(-\Delta H / k_{B} T\right)(\Delta H$ is the change in enthalpy) and $m$ is the particle mass. Thus we have $j \propto$ $\exp \left(-\Delta H / k_{B} T\right) / \sqrt{T}$ and taking the derivative on both sides lead to:

$$
\frac{d j}{j}=\left(\frac{\Delta H}{k_{B} T}-\frac{1}{2}\right) \frac{d T}{T}=\alpha \frac{d T}{T}
$$

$\alpha$ is expected to range between 10 and $60,{ }^{32}$ so even $0.1 \%$ variation in temperature can result in $1 \%$ variation in flux. Thus the Mg BEP appears to vary exponentially with $\mathrm{Mg}$ effusion cell temperature.

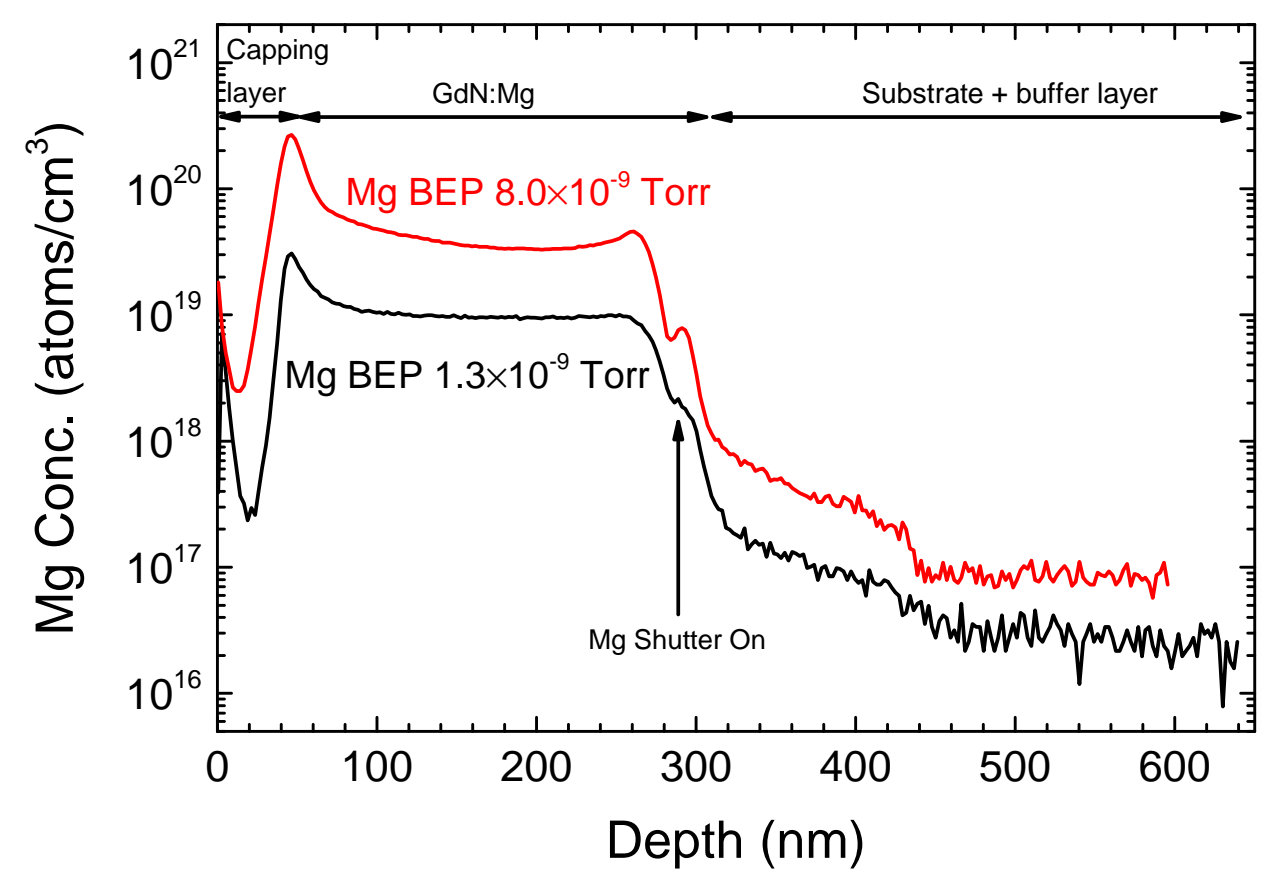

Figure 2.5: $\mathrm{Mg}$ concentration as a function of film depth for two Mg-doped GdN samples SCO917 and SCO919, measured using SIMS by OFC-Analytik in Germany. 
Secondary ion mass spectrometry (SIMS) was used to measure the Mg concentration of the Mg-doped GdN films, which was carried out by OFCAnalytik in Germany. From Figure (2.5), we find that GdN layers grown with $\mathrm{Mg}$ BEP $1.3 \times 10^{-9}$ and $8.0 \times 10^{-9}$ Torr (corresponding to $\mathrm{Mg}$ effusion cell temperature of 200 and $253{ }^{\circ} \mathrm{C}$ respectively) have $\mathrm{Mg}$ concentration of about $1 \times 10^{19}$ and $5 \times 10^{19}$ atoms $/ \mathrm{cm}^{3}$ respectively. The $\mathrm{Mg}$ concentration is reasonably constant throughout the GdN layer, indicating constant $\mathrm{Mg}$ incorporation rate during the growth. The measured $\mathrm{Mg}$ concentrations were used to estimate the $\mathrm{Mg}$ concentration in other samples with different $\mathrm{Mg}$ BEP values, by extrapolation. The upturn at the film surface is believed to be caused by the incorporation of residual $\mathrm{Mg}$ in the chamber prior to the deposition of the capping layer. The measurements also display low concentration of $\mathrm{Mg}$ atoms present in the AlN buffer layer for both samples, but it is difficult to think that $\mathrm{Mg}$ was actually incorporated in the layer because the AlN layer was grown in an MBE system that has never been in contact with Mg. Dr Markus Deimel from OFC-Analytik suggested that special effects during the sputtering process for SIMS (e.g. resputtering from the crater rim, memory effects) may be the cause for small $\mathrm{Mg}$ signals in the substrate, but he believes that the observed $\mathrm{Mg}$ concentration in the buffer layer is too high to be caused by the mentioned effects.

\subsection{Structural Properties}

In situ RHEED observations confirm the epitaxial character of the grown GdN thin films. ${ }^{26}$ Figure (2.6) shows RHEED patterns recorded after the growth of about $25 \mathrm{~nm}$ of $\operatorname{GdN}$ on $\operatorname{AlN}(0001)$, along (a) the [1-210] and (b) the [1-100] azimuths of AlN(0001). Both pictures display aligned diffraction spots which indicate that the films are epitaxial. Note that double spots (indicated by the arrows) along the [1-210] azimuth are not observable along the [1-100] azimuth, which is characteristic of twinned domains in the FCC structure of GdN.9,33

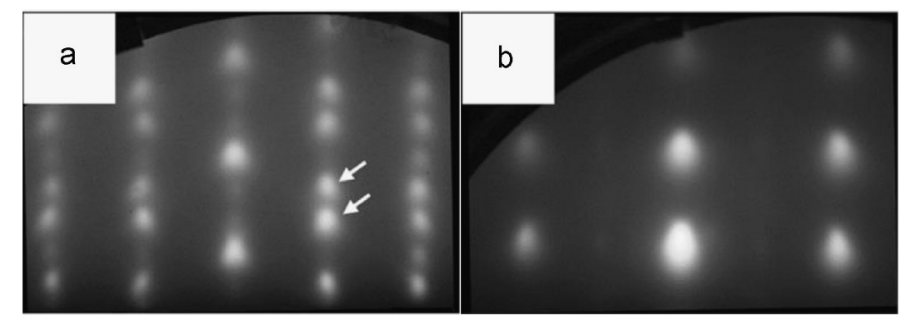

Figure 2.6: RHEED patterns recorded after the growth of about $25 \mathrm{~nm}$ of GdN on $\operatorname{AlN}(0001)$, along (a) the [1-210] and (b) the [1-100] azimuths of $\operatorname{AlN}(0001)$. 
The x-ray diffraction (XRD) technique allowed the investigation of the structural properties of GdN thin films. The "X'Pert PRO" x-ray diffraction system from PANalytical was used to assess the crystallographic properties of the grown samples. The copper $\mathrm{K}-\alpha$ radiation was used in the diffractometer, which has wavelength $\lambda=1.54 \AA$. X-ray diffraction occurs if the $\mathrm{x}$-ray incident on the sample has wavelength close to the lattice plane spacing $d_{h k l}$, where $h, k$ and $l$ are the Miller indices that describe the orientation of the lattice plane. If the diffracted x-rays satisfy the Bragg condition

$$
\lambda=2 d_{h k l} \sin \theta
$$

where $\theta$ is the angle between the incident x-rays and the scattering plane, they undergo constructive interference. Figure (2.7) shows that the incident angle $(\omega)$ is the angle between the x-ray source and the sample, and the diffracted angle $(2 \theta)$ is the angle between the incident $x$-ray beam and the detector angle. $2 \theta$-scans are plots of intensity as a function of diffracted angle, obtained by fixing the sample and only varying the detector position. It gives access to the texture, lattice parameter, residual stress etc. of the sample. Rocking curves are plots of intensity as a function of incident angle, obtained by fixing the detector at an expected Bragg reflection angle and measuring the diffracted x-ray intensity as the sample is rotated. Rocking curves give access to information about imperfections in the sample, such as the curvature or the tilt.

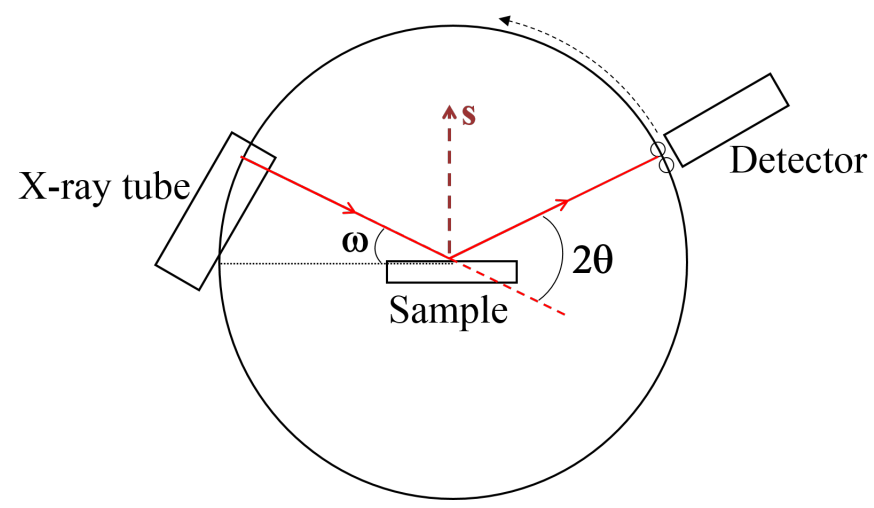

Figure 2.7: A diagram illustrating the arrangement of the XRD system and the angles involved. 


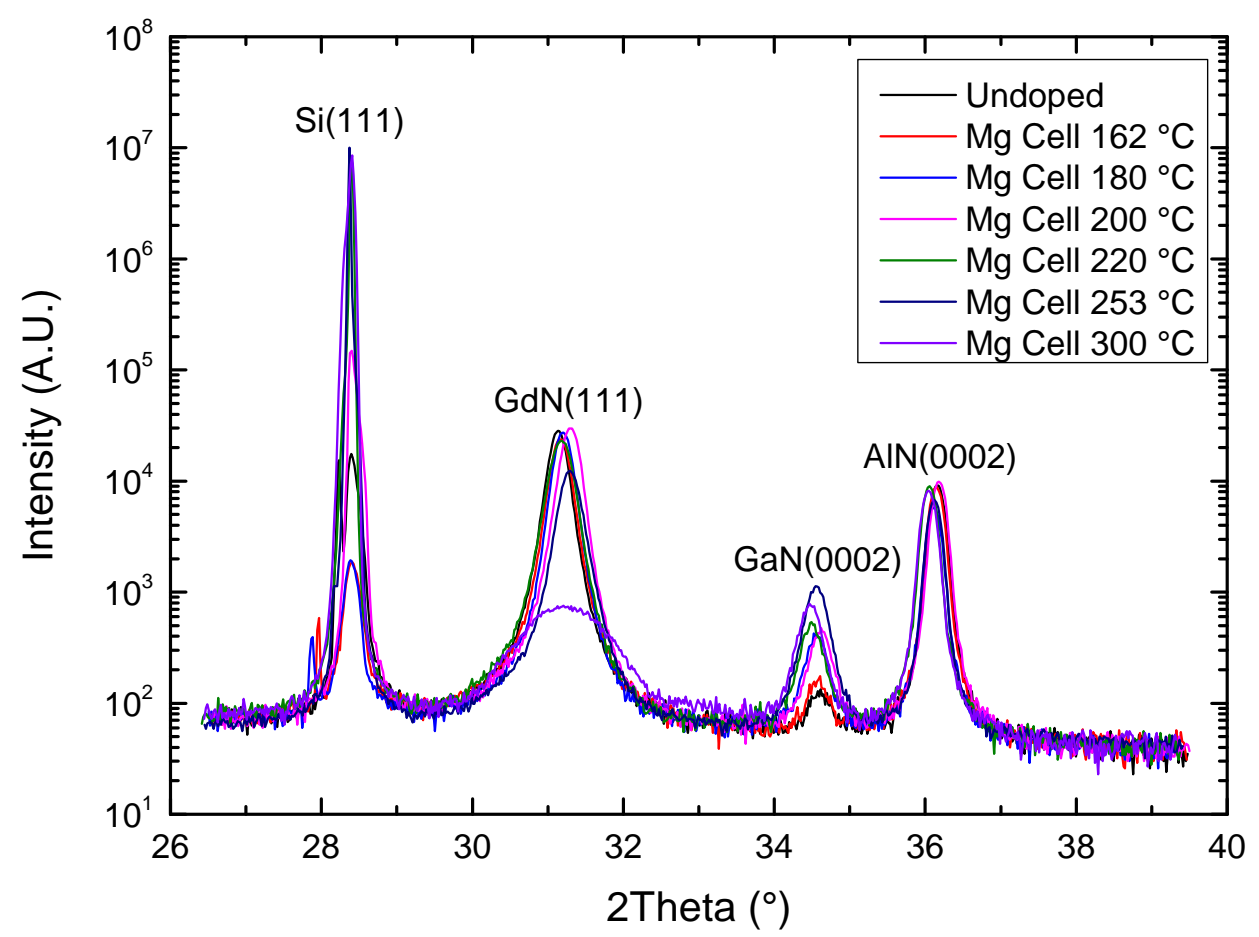

Figure 2.8: 20-scan of Mg-doped epitaxial GdN thin films with different Mgdoping levels. The plot has been normalised using the $\mathrm{Si}(111)$ peak as a reference so that all the $\mathrm{Si}(111)$ peaks from different films coincide at $28.42^{\circ}$.

Figure (2.8) displays the $2 \theta$-scan of epitaxial GdN films with different $\mathrm{Mg}$ doping levels. The most prominent peak at $\sim 28.42^{\circ}$ is due to the $\mathrm{Si}(111)$ substrate, and the peaks at $\sim 34.5^{\circ}, \sim 36^{\circ}$ are due to the $\mathrm{GaN}(0002)$ capping layer, $\mathrm{AlN}(0001)$ buffer layer, respectively. Note that only the (111) reflection at $\sim 31.5^{\circ}$ is observed for $\mathrm{GdN}$, as favoured by the hexagonal AlN(0001) surface, ${ }^{12}$ independent of the Mg-doping. For all the films, reflections due to Gd metal such as $\operatorname{Gd}(100)$ peak at $\sim 29^{\circ}$ or $\operatorname{Gd}(101)$ peak at $\sim 33^{\circ}$ are unobserved. ${ }^{19}$ This indicates that there is no Gd cluster formation in all the films, and $\mathrm{Gd} / \mathrm{N}$ stoichiometry is not dramatically affected by the Mg-doping. However, a significant change in the crystal structure for the most heavily $\mathrm{Mg}$-doped film ( $\mathrm{Mg}$ cell temp. $300{ }^{\circ} \mathrm{C}$ ) is observed, as we can see from its widened and weakened $\operatorname{GdN}(111)$ peak. This corresponds to $\mathrm{Mg}$ concentration of $\sim 2 \times 10^{20}$ atoms $/ \mathrm{cm}^{3}$ incorporated in the GdN layer, obtained by extrapolating the SIMS results from Figure (2.5). 


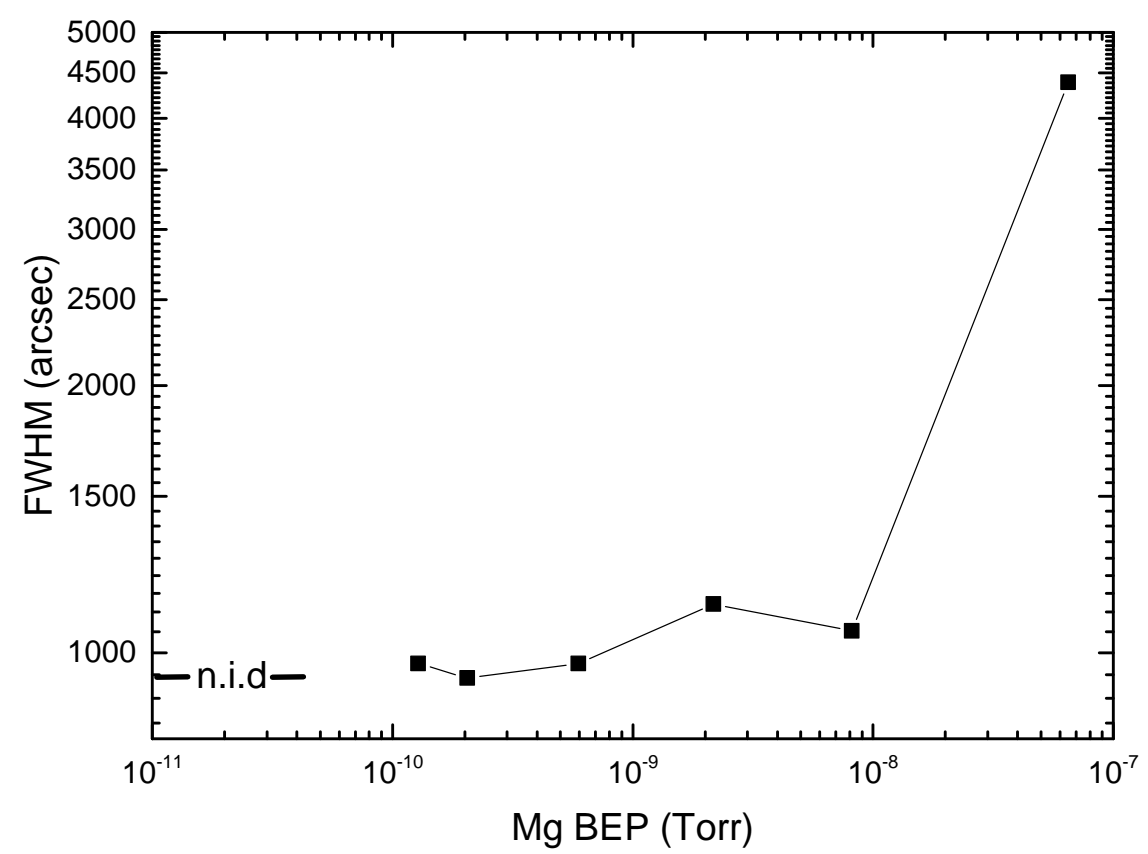

Figure 2.9: FWHM values of $\operatorname{GdN}(111)$ peaks from the $2 \theta$-scan as a function of Mg BEP for GdN films with different Mg-doping levels. The "n.i.d" line indicates the FWHM value for the undoped GdN film.

Figure (2.9) shows that there is no clear decrease in the crystalline quality of GdN films with increasing Mg-doping level, for Mg BEP up to $\sim 8.2 \times 10^{-9}$ Torr. Taking the full width at half maximum (FWHM) of GdN(111) peak from Figure (2.8), we see that the undoped film has FWHM of $\sim 900$ arcsec, and this value remains similar for the lightly Mg-doped films. Even for a more heavily Mg-doped film (Mg BEP $\sim 2.2 \times 10^{-9}$ Torr) the FWHM only increases to $\sim 1100$ arcsec which is still not very significant. However, for the most heavily Mg-doped film (Mg BEP $\sim 6.5 \times 10^{-8}$ Torr $)$, the FWHM is dramatically increased to $\sim 4400$ arcsec which clearly indicates a substantial deterioration in the crystalline quality. 


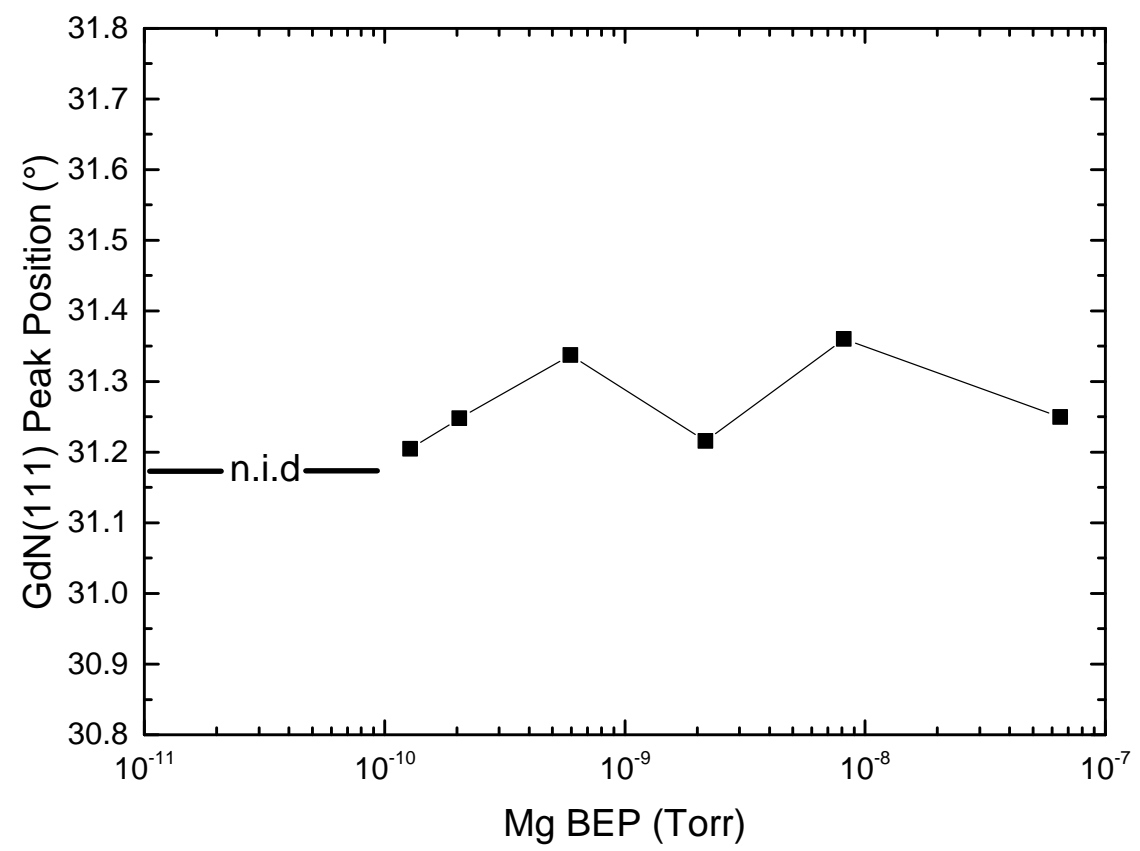

Figure 2.10: The angle of $\operatorname{GdN}(111)$ reflection from the $2 \theta$-scan as a function of Mg BEP for GdN films with different Mg-doping levels. The "n.i.d" line indicates the reflection angle for the undoped GdN.

The lattice spacing between adjacent $(h k l)$ planes are giving by $d_{h k l}=$ $a / \sqrt{h^{2}+k^{2}+l^{2}}$, and this can be combined with Bragg's law to obtain the lattice constant $(a)$ of a cubic system.

$$
a=\frac{\lambda \sqrt{h^{2}+k^{2}+l^{2}}}{2 \sin \theta}
$$

With the observed GdN(111) peak positions in Figure (2.10) for films with various Mg-doping levels, Equation (2.4) can be used to estimate the outof-plane lattice constant. The lattice constant for the undoped film is 4.963 $\AA$, which is in good agreement with the reported value of $4.974 \AA$ in the literature. ${ }^{1}$ The value for the most heavily Mg-doped film is $\sim 4.95 \AA$ which is still quite close to the reported value. So unlike the FWHM, the peak position is not significantly affected even for the heavily-doped GdN. Thus no systematic variation in the out-of-plane lattice constant with increasing Mg-doping level is observed. 


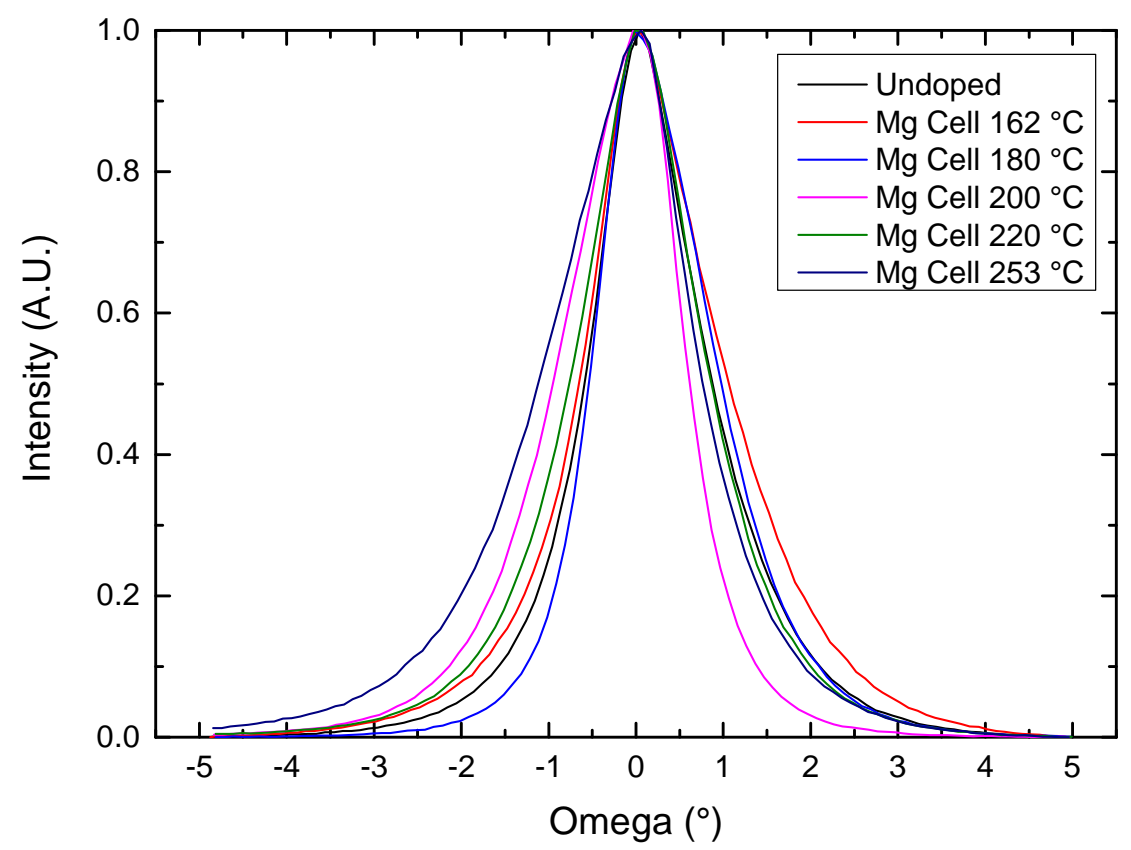

Figure 2.11: Rocking curves of the GdN(111) plane for films with different Mgdoping levels. The intensity has been normalised for easier comparison.

It can be seen in Figure (2.11) that the rocking curves of GdN films do not show an apparent systemic variation with increasing Mg-doping level, similarly to the $2 \theta$-scan. The FWHM values range from 5500 arcsec to 7150 arcsec (from undoped to $\mathrm{Mg}$ cell temp. $253^{\circ} \mathrm{C}$ ), which indicates the films have significant mosaic spread, ${ }^{34}$ but there is no systemic variation with increasing level of Mg-doping, as can be seen in Figure (2.12). However, the rocking curve of the most heavily $\mathrm{Mg}$-doped film at $\mathrm{Mg}$ effusion cell temp. $300{ }^{\circ} \mathrm{C}$ (not shown due to extremely weak signal), corresponding to $\mathrm{Mg}$ concentration $\sim 2 \times 10^{20}$ atoms $/ \mathrm{cm}^{3}$, showed XRD intensity orders of magnitude smaller than the rest of the films and its FWHM significantly broadened, indicating a substantial deterioration in the crystalline quality. 


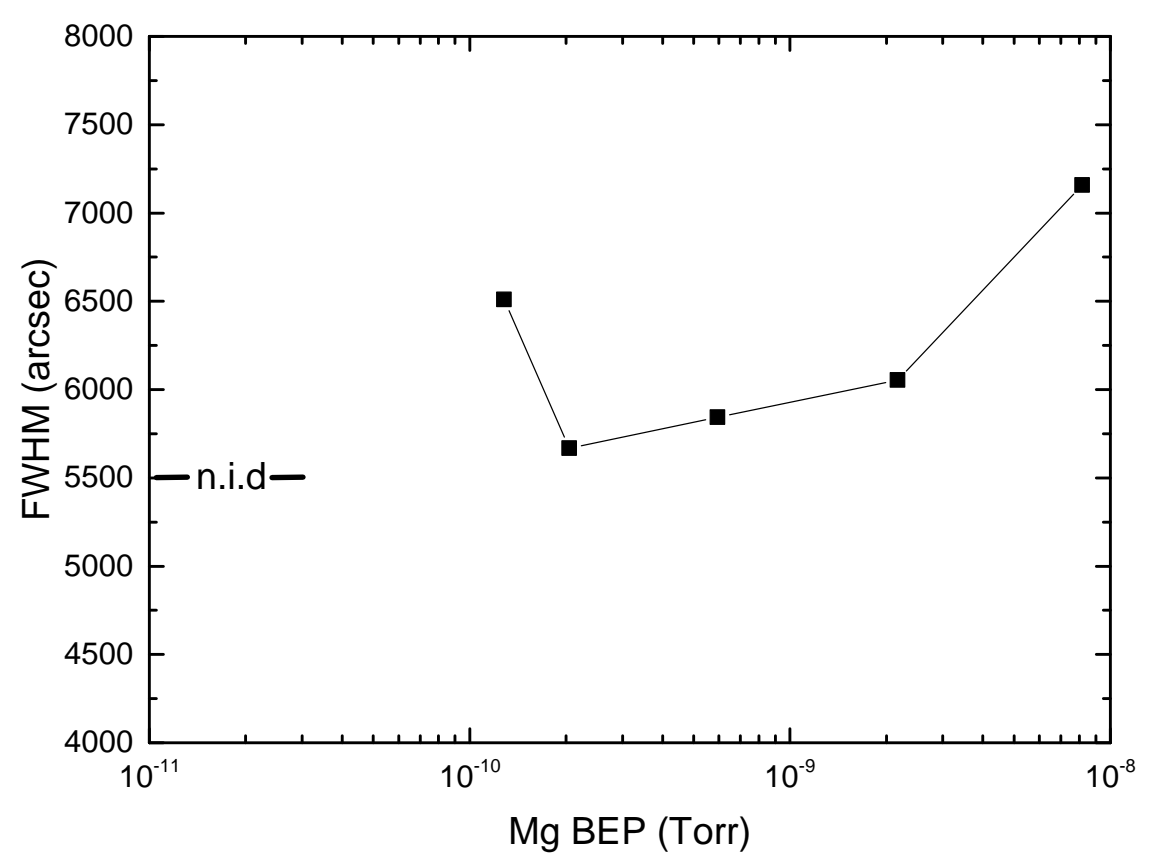

Figure 2.12: FWHM values of rocking curves as a function of Mg BEP for GdN films with different Mg-doping levels. The "n.i.d" line indicates the FWHM value for the undoped GdN film.

Overall, there is no significant systematic variation in the crystallographic structure of Mg-doped epitaxial GdN thin films, for $\mathrm{Mg}$ concentrations up to $\sim 5 \times 10^{19}$ atoms $/ \mathrm{cm}^{3}$. However, from an $\mathrm{Mg}$ concentration of $\sim 2 \times 10^{20}$ atoms $/ \mathrm{cm}^{3}$, a clear deterioration in the crystalline quality was observed. This issue is similar to that of GaN in some aspects, because the quality of a GaN layer is degraded with incorporation of such a high $\mathrm{Mg}$ concentration. It has been reported that Mg-doped epitaxial GaN thin films grown by MBE show a significant increase in density of non-radiative defects with $\mathrm{Mg}$ concentration $\sim 2 \times 10^{20}$ atoms $/ \mathrm{cm}^{3},{ }^{35}$ and $\mathrm{Mg}$-doped GaN films grown by metal organic chemical vapour deposition (MOCVD) display a dramatic decrease in crystalline quality once its $\mathrm{Mg}$ concentration is above $\sim 10^{20}$ atoms $/ \mathrm{cm}^{3}$, due to the formation of Mg clusters. ${ }^{36}$ 


\section{Chapter 3}

\section{Electrical and Magnetic Properties}

\subsection{Electrical Characterisation Techniques}

The resistivity of Mg-doped epitaxial GdN thin films were measured using the four-point probe technique. This technique has the advantage of being able to measure the resistance of a sample even if there are insulating components between the probes and the sample. ${ }^{37}$ The sample was mounted on top of a copper block with four probes, where each probe touches the sample and acts as a terminal, as shown in Figure (3.1). A small drop of silver conductive paint was put between each probe and the sample, to provide better contacts. An electrically insulating (but thermally conducting) thin paper was placed between the sample and the copper block to prevent the formation of a short circuit. Thermal grease was applied between the sample and the copper block to ensure good thermal conduction. The copper block was placed inside a helium-cooled cryostat so that the sample resistivity could be measured at a temperature as low as $4 \mathrm{~K}$, allowing temperaturedependent measurements. The sample is under vacuum $\left(<1 \times 10^{-4}\right.$ Torr $)$ and in dark while it is inside the cryostat.

Van der Pauw geometry was used for the resistivity measurements (shown in Figure (3.1)) as it has the advantage of shape-independent measurement of sample resistance, provided these four conditions are satisfied: (1) sample has uniform thickness, (2) the contacts are small compared to the surface area of the sample, (3) the contacts are placed at the circumference of the sample, (4) the sample has no isolated holes. If the conditions are met, the 


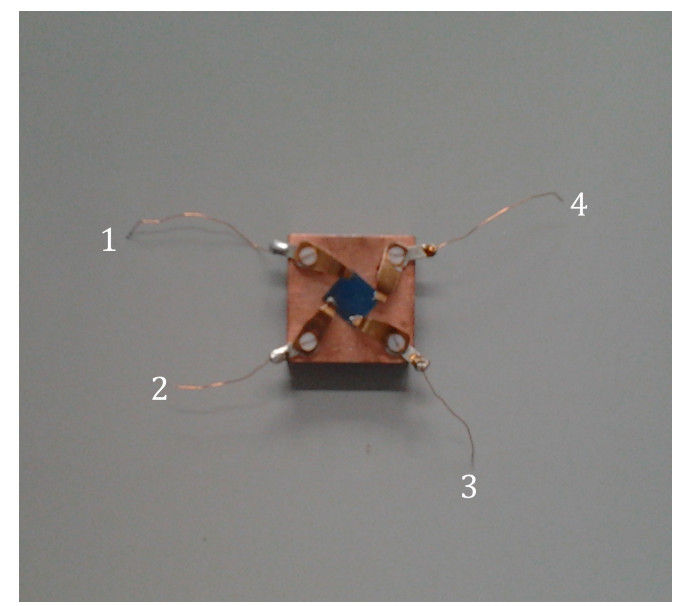

Figure 3.1: A sample of size $1 \mathrm{~cm} \times 1 \mathrm{~cm}$ is mounted on the copper block. The contacts arranged in van der Pauw geometry.

resistivity $(\rho)$ of a square-shaped thin film is given by

$$
\rho=\frac{\pi}{\ln (2)} d R_{12,34}
$$

where $d$ is the thickness of the film and $R_{12,34}=V_{34} / I_{12}$ is the resistance obtained from $I_{12}$ and $V_{34} \cdot{ }^{37}$ Here, $I_{12}$ is the current from contact 1 to contact 2 and $V_{34}$ is the potential difference between contacts 3 and 4 .

Hall effect measurements were performed on GdN thin films using the fourpoint probe technique and van der Pauw geometry, similar to the resistivity measurement. A magnetic field of strength $0.9 \mathrm{~T}$ was applied perpendicular to the sample surface and the Hall voltage $\left(V_{H}\right)$ was measured, which is related to the carrier concentration by the following relation

$$
V_{H}=\frac{I B}{n q d}
$$

where $I$ is the current, $B$ is the magnetic field strength, $n$ is the carrier concentration, $q$ is the charge of the carrier, and $d$ is the thickness of the GdN film. Here, $V_{H}=V_{13}$ and $I=I_{24}$ such that the direction of the current flow is perpendicular to the path between the two contacts whose potential difference is measured. Since the values of $I, B, q$ and $d$ are all known, the carrier sign and concentration can be calculated from the measured Hall voltage. 


\subsection{Electrical Properties}

Encouraged by the promising growth results, resistivity and Hall effect measurements were performed at room temperature.

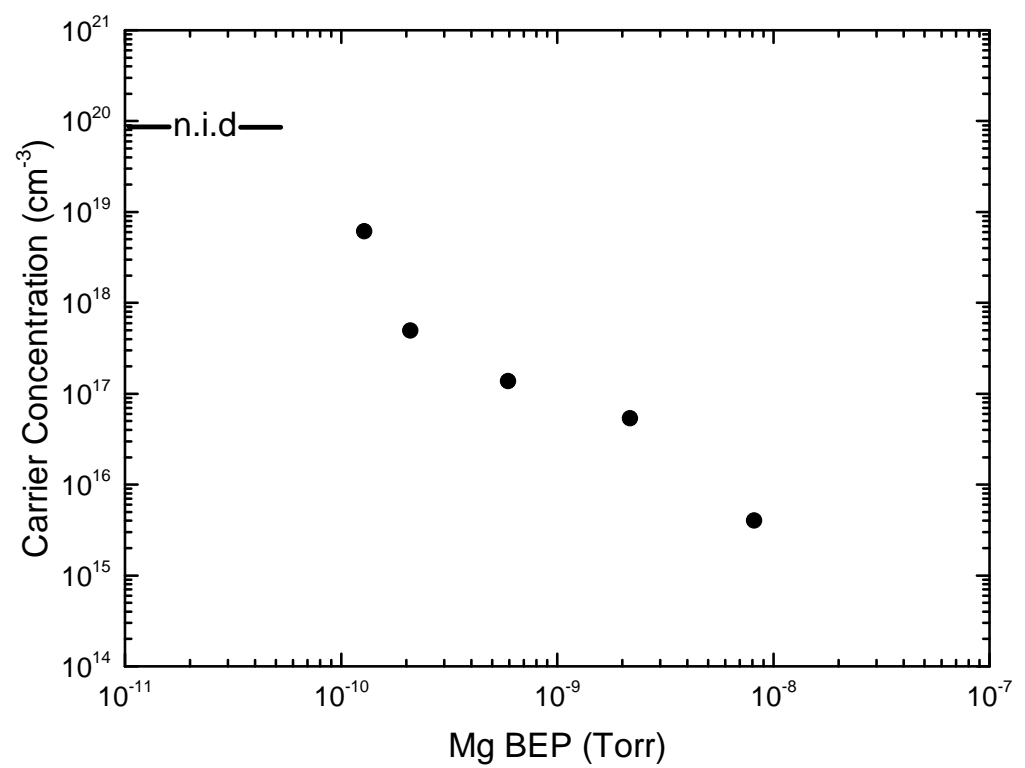

Figure 3.2: Carrier concentration at room temperature as a function of Mg BEP during the growth. The "n.i.d." line indicates the carrier concentration of the GdN film with no intentional Mg-doping.

From the room temperature Hall effect measurement, it was found that all of the films had n-type free carriers, i.e. electrons. Figure (3.2) displays a strong reduction in carrier concentration with increasing $\mathrm{Mg}$-doping level. The n-type carrier concentration of $7 \times 10^{20} \mathrm{~cm}^{-3}$ for an undoped GdN film (indicated by the n.i.d line) can be reduced by as much as five orders of magnitude down to $\sim 5 \times 10^{15} \mathrm{~cm}^{-3}$ for a heavily doped GdN film. Thus electron compensation in $\mathrm{GdN}$ via incorporating $\mathrm{Mg}$ is clearly seen over a full range of dopant concentrations. Note that the carrier concentration of $7 \times 10^{20} \mathrm{~cm}^{-3}$ in the undoped film corresponds to $\sim 0.02$ electrons per primitive cell, which suggests $V_{N}$ concentration less than $\sim 1 \%$, assuming that each $V_{N}$ donates a single free electron. 


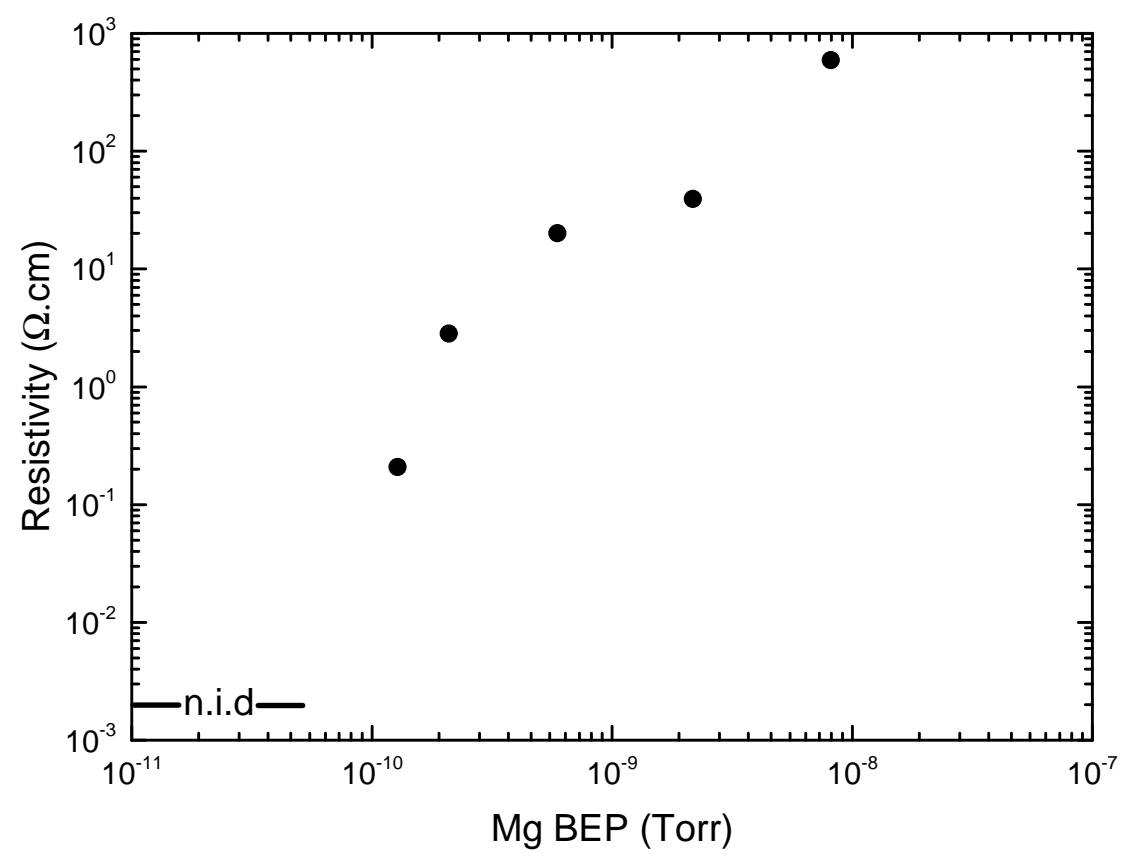

Figure 3.3: Resistivity at room temperature as a function of $\mathrm{Mg}$ BEP during the growth. The "n.i.d." line indicates the resistivity of the GdN film with no intentional Mg-doping.

Room temperature resistivity measurements reveal a substantial change in the resistivity for samples with different Mg-doping levels. A clear increase in resistivity due to increasing Mg-doping level can be seen in Figure (3.3). The undoped GdN film is quite conductive with a resistivity of $0.002 \Omega \mathrm{cm}$, whereas a heavily Mg-doped GdN film with $\mathrm{Mg}$ concentration $\sim 5 \times 10^{19}$ atoms $/ \mathrm{cm}^{3}$ has a resistivity of $600 \Omega \mathrm{cm}$, which is over five orders of magnitude change. 


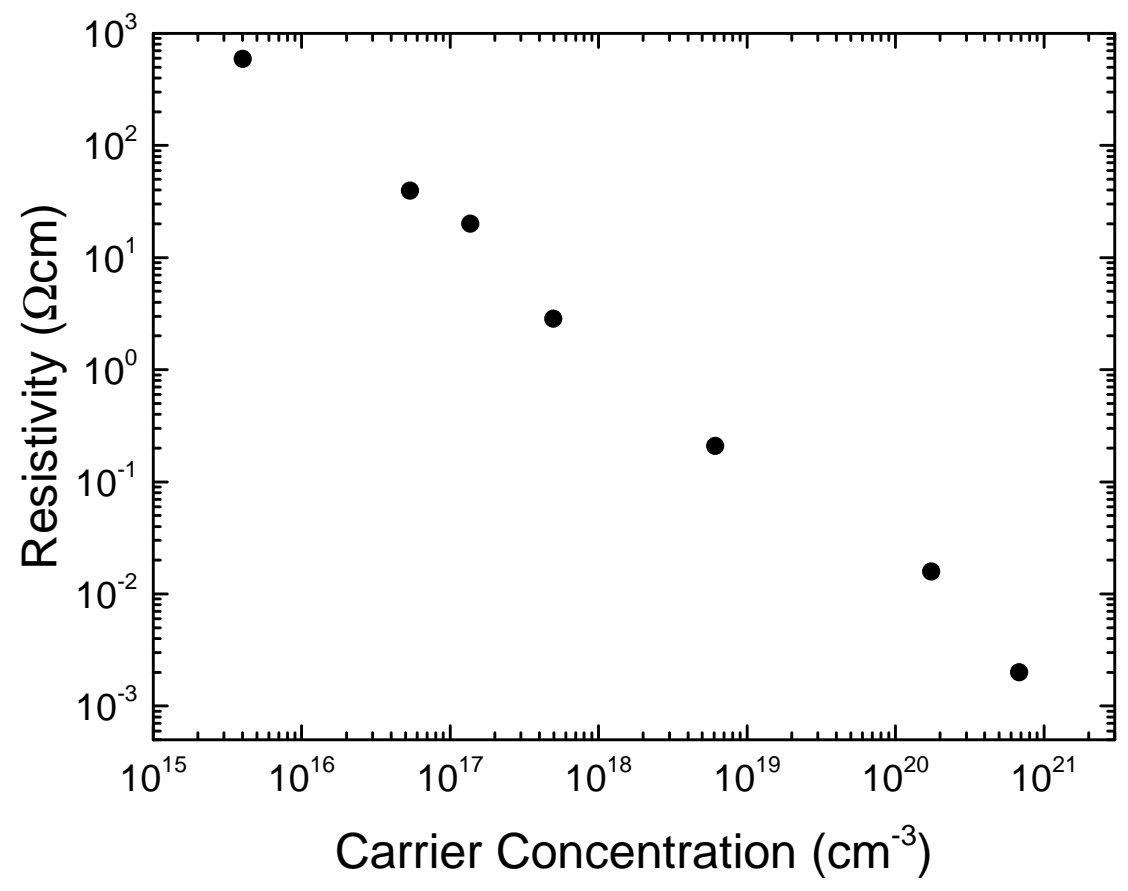

Figure 3.4: Resistivity as a function of carrier concentration at room temperature for GdN films with different Mg-doping levels.

Figure (3.4) shows that the resistivity varies inversely proportional to the carrier concentration for over five orders of magnitude at room temperature, thus indicating that the electron mobility remains quite constant over the full range of $\mathrm{Mg}$ concentrations in GdN. From the y-intercept of the linear trend in Figure (3.4), we obtain electron mobility of $\sim 5 \mathrm{~cm}^{2} / \mathrm{Vs}$ for the Mg-doped GdN films independent of the Mg-doping level.

One thing to notice from the above results is that a $\mathrm{Mg}$ concentration of $\sim 10^{19} \mathrm{~cm}^{-3}$ passivates an electron concentration of $\sim 10^{21} \mathrm{~cm}^{-3}$ via compensation, which is difficult to understand. If it was simply a single electron donated by a $V_{N}$ being accepted by a single $\mathrm{Mg}^{2+}$ ion in a one-to-one manner, such a large change in carrier concentration would not have been seen. It is more likely to be due to some change in the network by the introduction of $\mathrm{Mg}$ atoms such that $V_{N}$ concentration is forced to decrease. Also, there is recent evidence that the electrons are found in shallow traps near the $V_{N}$, forming magnetic polarons in an extended-state impurity band for $V_{N}$ concentrations above $\sim 10^{20} \mathrm{~cm}^{-3}{ }^{18}$ This will be discussed further in the next section with the magnetic data. 
Now, I will focus on three Mg-doped epitaxial GdN thin films with following growth conditions and properties. For convenience, the samples are named according to their carrier concentrations at room temperature, where $\mathrm{H}, \mathrm{M}$ and L indicate high, moderate and low carrier concentration, respectively.

\begin{tabular}{|l|l|l|l|l|} 
Name & Sample & Mg Flux (Torr) & $\begin{array}{l}\text { Carrier Conc. } \\
\left(\mathrm{cm}^{-3}\right)\end{array}$ & $\begin{array}{l}\text { Resistivity } \\
(\Omega \mathrm{cm})\end{array}$ \\
\hline $\mathrm{H}$ & SCO910 & 0 & $\sim 7 \times 10^{20}$ & $\sim 0.0023$ \\
\hline M & SCO981 & $1.28 \times 10^{-10}$ & $\sim 6 \times 10^{18}$ & $\sim 0.2$ \\
\hline L & SCO988 & $5.94 \times 10^{-10}$ & $\sim 1 \times 10^{17}$ & $\sim 20$ \\
\hline
\end{tabular}

Figure 3.5: Samples used for temperature-dependent resistivity measurements. The carrier concentration and resistivity are room temperature values.

Figure (3.6) shows how the temperature-dependent resistivity of GdN films changes with increasing $\mathrm{Mg}$-doping level. It is clear that reduction in carrier concentration via Mg-doping applies for temperatures ranging from room temperature down to $4 \mathrm{~K}$. As we move from sample $\mathrm{H}$ to $\mathrm{M}$ and then to $\mathrm{L}$, there is two orders of magnitude increase in resistivity each time. There are two main features to note here.

First, we look at the temperature coefficient of resistance (TCR) from room temperature down to $\sim 150 \mathrm{~K}$. For the undoped sample $\mathrm{H}$, the TCR above $\sim 150 \mathrm{~K}$ is positive, which is reminiscent of metallic behaviour, as commonly seen in heavily electron-doped semiconductors. Clearly, the 0.02 electrons per primitive cell form a degenerate electron gas, though it is unclear whether these electrons reside in the conduction band or in a defect-centred tail below the conduction band edge. In contrast, the TCR above $\sim 150 \mathrm{~K}$ is slightly negative for the moderately Mg-doped sample M, implying more intrinsic semiconducting behaviour. Furthermore, the heavily Mg-doped sample L not only has a semiconducting magnitude of resistance but also shows a strong negative TCR above $150 \mathrm{~K}$, which is typical of a semiconductor but with a small activation energy. Thus it is clear that Mg-doping causes these GdN films to behave more like intrinsic semiconductors.

Another important feature to note is the shift in the resistivity anomalies. For sample $\mathrm{H}$, the anomaly peaks near $70 \mathrm{~K}$ which is very close to its Curie temperature $\left(T_{C}\right)$ as we will see in the next section. However, the anomaly peaks at a lower temperature near $60 \mathrm{~K}$ for sample $\mathrm{M}$, and the anomaly is further shifted down for sample L, peaking near $55 \mathrm{~K}$, which are also close to the $T_{C}$ of each sample (shown in next section). Thus there is some 


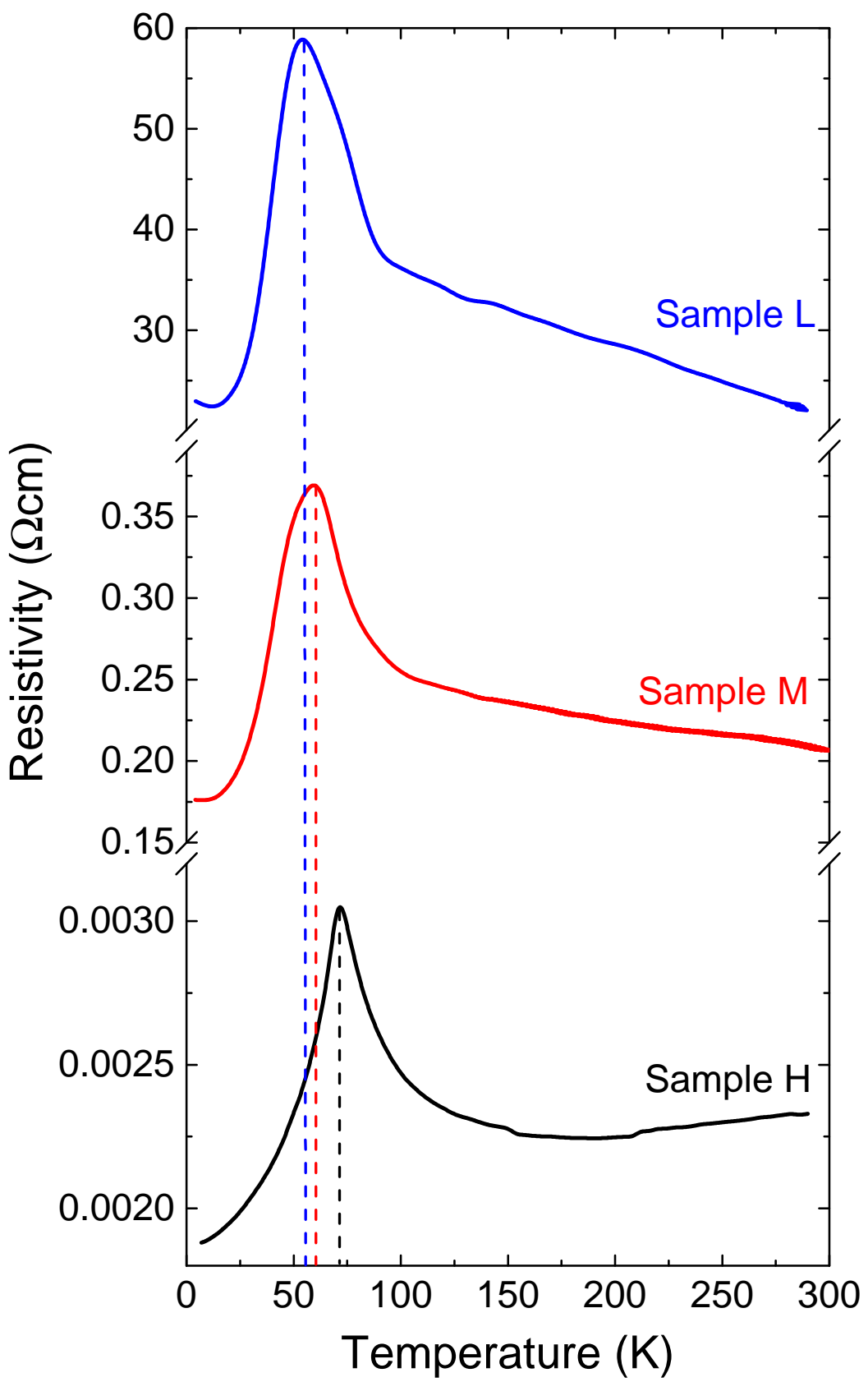

Figure 3.6: Temperature-dependent resistivity of GdN films with different $\mathrm{Mg}$ doping levels. 
correlation between the peak shifting and carrier concentration. There are at least two competing contributions that causes the resistivity anomaly in GdN. One is temperature-dependence of carrier concentration. At $T_{C}$, the exchange field splits the conduction band such that the majority spin state is pulled down and minority spin state is pushed up. ${ }^{5,11,12}$ This causes reduction in the band gap, resulting in more carriers and reducing the resistivity. Another contribution is due to magnetic disorder scattering, because magnetic fluctuations become stronger near $T_{C} \cdot{ }^{18}$ Below $T_{C}$, more electrons are scattered due to magnetic disordering, which would increase the resistivity. This indicates a link between charge carriers and the magnetic exchange mechanism, and it will be discussed further in the upcoming section along with the magnetic data.

Also, note that after the peaking, at the lowest temperatures, the TCR becomes similar to the behaviour above $150 \mathrm{~K}$. This is easily seen by the flattening near $10 \mathrm{~K}$ for sample $\mathrm{M}$ and the strong negative TCR below 10 $\mathrm{K}$ for sample $\mathrm{L}$. Thus for sample $\mathrm{L}$, its ferromagnetic phase is also semiconducting.

\subsection{Magnetic Characterisation Techniques}

The magnetic characterisation of Mg-doped epitaxial GdN thin films was carried out using the "Magnetic Property Measurement System" (MPMS) from Quantum Design. A schematic diagram of the MPMS is shown in Figure (3.7). This system is able to measure very small magnetic fields through the use of a superconducting quantum interference device (SQUID). It is able to apply a strong magnetic field as large as $7 \mathrm{~T}$, using a superconducting helium-cooled magnet. The system is helium-cooled and the measurements can be done at a temperature as high as $400 \mathrm{~K}$, down to $2 \mathrm{~K}$. The field direction was parallel to the sample surface so that in-plane magnetisation was induced.

For a temperature-dependent magnetisation measurement, there are two different ways to make measurements. One is a zero-field-cooled (ZFC) measurement, where the sample (initially above its Curie temperature) is cooled down to $2 \mathrm{~K}$ without the presence of any applied magnetic field. Then a constant field is applied to the sample and its magnetisation is measured while it is warming up. Another is field-cooled (FC) measurement, where the sample (initially above its Curie temperature) is cooled down to $2 \mathrm{~K}$ in the presence of a constant applied magnetic field and its magnetisation 


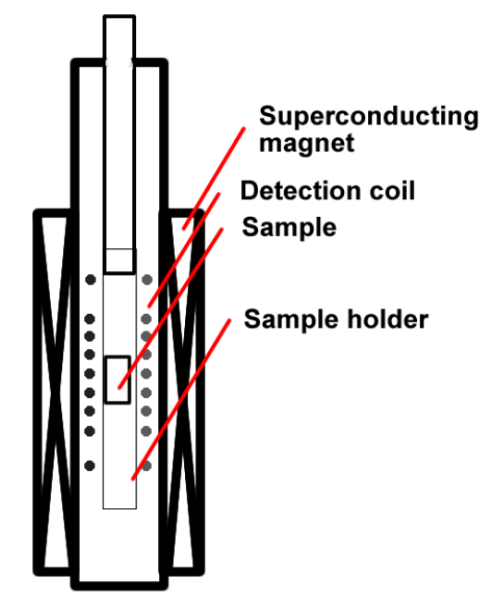

Figure 3.7: A schematic diagram of the MPMS.

is measured while it is cooling down. Because the sample shows hysteresis below its Curie temperature, the magnetisation is dependent on its magnetic prehistory. So these two different measuring methods do not necessarily give the same results, unless the applied magnetic field is large enough to magnetise the sample completely for all values of temperature.

A field-dependent magnetisation measurement is done by sweeping the applied magnetic field from $4 \mathrm{~T}$ to $-4 \mathrm{~T}$ (same field strength but now in the opposite direction) then back to $4 \mathrm{~T}$. Such sweeping gives us a "hysteresis loop" which gives us information about the magnetic properties of the sample such as the saturation magnetisation and coercive field. Saturation magnetisation is the maximum induced magnetic moment that can be obtained in an applied magnetic field. Coercive field is the magnetic field strength at which the magnetisation is reduced from saturation to zero during the sweeping. 


\subsection{Magnetic Properties}

The characterisation was carried out on following samples of epitaxial GdN thin films with different Mg-doping levels, shown in Figure (3.8). Again, they were named according to their carrier concentrations for convenience.

\begin{tabular}{|l|l|l|l|} 
Name & Sample & $\begin{array}{l}\text { Mg Conc. } \\
\left(\mathrm{cm}^{-3}\right)\end{array}$ & $\begin{array}{l}\text { Carrier Conc. At } \\
\text { Room Temp. }\left(\mathrm{cm}^{-3}\right)\end{array}$ \\
\hline $\mathrm{H}$ & SCO910 & 0 & $\sim 7 \times 10^{20}$ \\
\hline M & SCO917 & $1 \times 10^{19}$ & $\sim 5 \times 10^{16}$ \\
\hline L & SCO919 & $5 \times 10^{19}$ & $\sim 6 \times 10^{15}$ \\
\hline
\end{tabular}

Figure 3.8: Samples used for magnetic measurements.

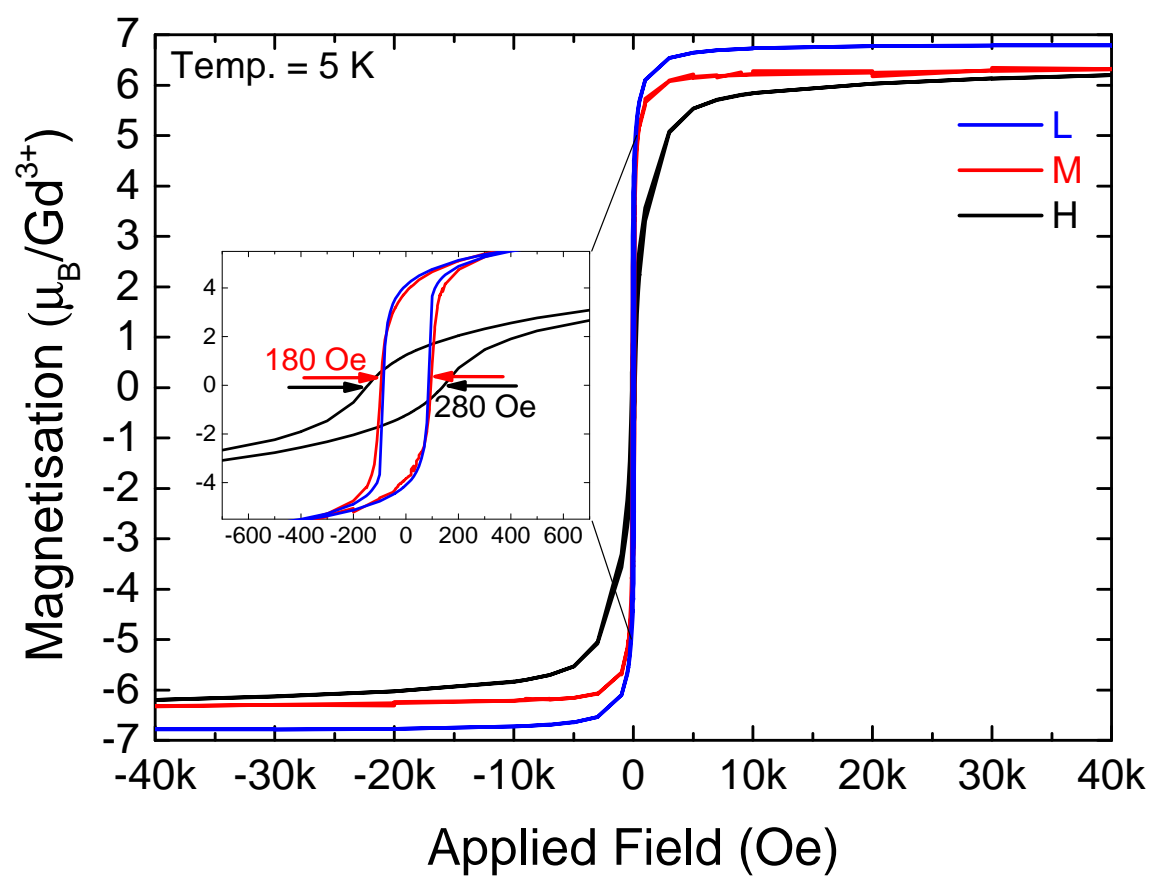

Figure 3.9: Field-dependent magnetisation of GdN films with different Mg-doping levels at $5 \mathrm{~K}$. The applied field was in-plane.

The field-dependent magnetisation measurements of Mg-doped GdN films in Figure (3.9) show saturation magnetisation $\left(M_{\text {sat }}\right)$ of $6.2 \mu_{B}, 6.3 \mu_{B}$ and 
$6.8 \mu_{B}$ per $\mathrm{Gd}^{3+}$ for sample $\mathrm{H}, \mathrm{M}$ and $\mathrm{L}$, respectively. The obtained values are close to the $7 \mu_{B}$ per $\mathrm{Gd}^{3+}$ for a half-filled $4 f$ shell as predicted by Hund's rule, and in agreement with values reported in the literature. ${ }^{7,16,17}$ The $\mathrm{M}_{\text {sat }}$ is closer to $6 \mu_{B} / \mathrm{Gd}^{3+}$ for the sample $\mathrm{H}$, and it shows a slight tendency to become closer to $7 \mu_{B} / \mathrm{Gd}^{3+}$ as the carrier concentration decreases for GdN. Looking at the magnified plot, a clear magnetic hysteresis loop is observed, and we see that the coercive field is $\sim 140$ Oe for sample $\mathrm{H}$ and $\sim 90$ Oe (half the width of the loop) for both of the Mg-doped GdN films, M and L. Thus the coercive field is reduced for the Mg-doped samples, but the level of doping did not affect the amount of reduction in the coercive field, unlike the $M_{\text {sat }}$.

Ludbrook et al. suggested two possible competing mechanisms that could affect the coercive field in epitaxial GdN thin films grown by pulse laser deposition technique. ${ }^{17}$ One is due to the nucleation of reversal of magnetisations. The reversal of magnetisation can nucleate at localised magnetic inhomogeneities or defects, and since defects are more likely to be found in larger crystals, larger crystallite size leads to samples having lower coercive field. Another is that domain walls can be pinned by defects, which causes samples with larger crystallite volume to have higher coercive field. However, in the previous section we did not see a clear systematic change in crystalline quality with increasing Mg-doping level, thus the origin of change in coercive field with Mg-doping still remains difficult to explain.

The temperature-dependent FC magnetisation measurements in Figure (3.10) show that all GdN films exhibit ferromagnetic behaviour at low temperature, but with substantial contrasts. Clearly, we see an onset of ferromagnetism below the $T_{C}=70 \mathrm{~K}$ for all the samples. For sample $\mathrm{H}$, the ferromagnetic transition near $70 \mathrm{~K}$ is the only transition it goes through. Plotting the inverse of susceptibility against temperature for sample $\mathrm{H}$ shows its paramagnetic response above $70 \mathrm{~K}$, as shown by the linear fit in Figure (3.11). The concentration of magnetic atoms in sample $\mathrm{H}$ can be estimated by Curie-Weiss law:

$$
\chi=\frac{n \mu_{0} \mu_{B}^{2} g^{2}}{3 k_{B} T} J(J+1)
$$

where $\chi$ is the magnetic susceptibility, $g$ is the g-factor, $J$ is the total angular momentum number, and $n$ is the concentration of magnetic atoms. ${ }^{38}$ CurieWeiss law shows that $1 / \chi \propto T$ for paramagnetic materials, thus $n$ can be estimated from the gradient of the linear fit above $T_{C}$ in Figure (3.11). A calculation using $g=2$ and $J=7 / 2$ leads to $n=6.9 \times 10^{22} \mathrm{~cm}^{-3}$, and comparing this to the density of Gd atoms in GdN (typically $\sim 7 \times 10^{22}$ 


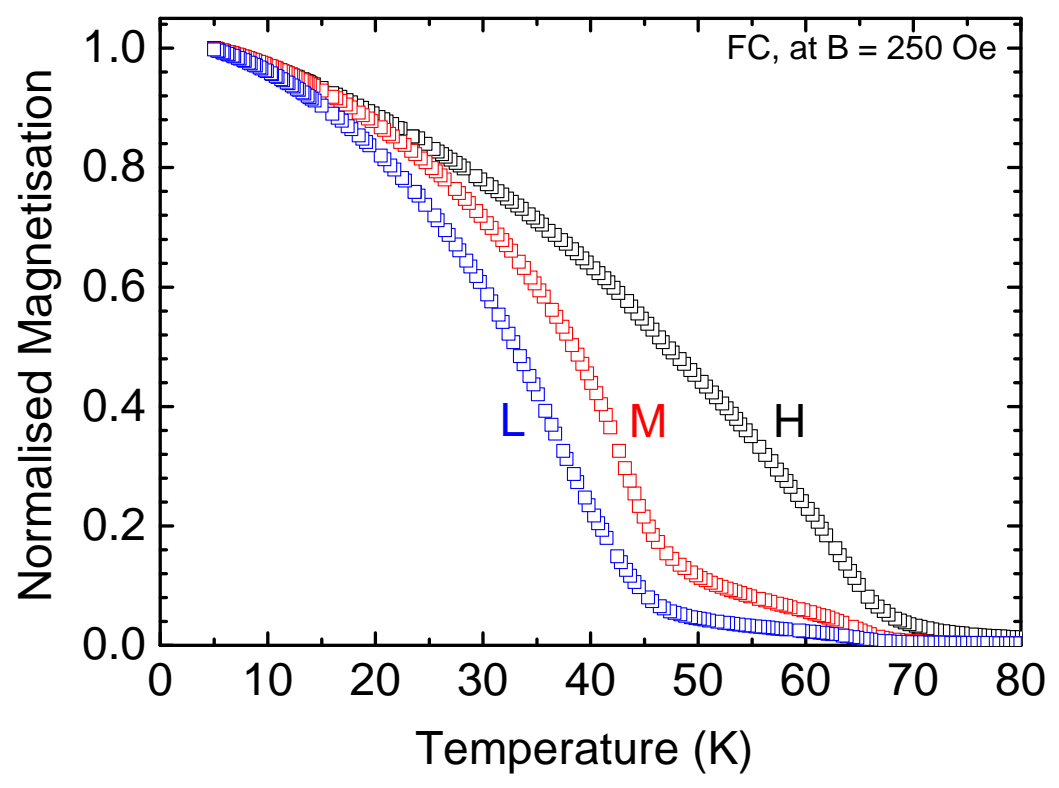

Figure 3.10: Temperature-dependent magnetisation of GdN films with different Mg-doping levels, field-cooled at 250 Oe. The magnetisation is normalised for easier comparison.

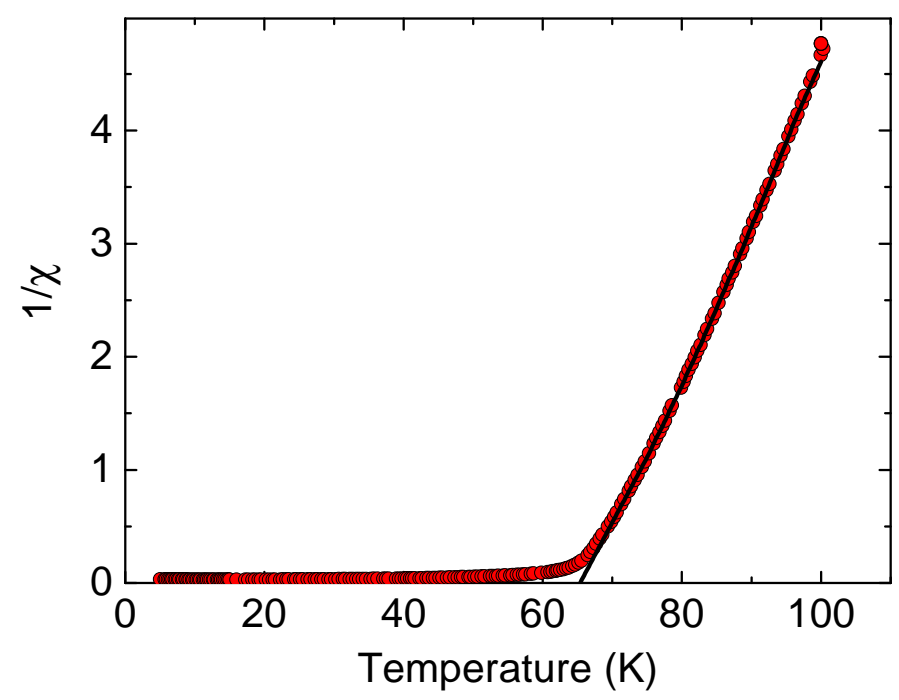

Figure 3.11: Inverse of susceptibility as a function of temperature for sample $\mathrm{H}$, showing its paramagnetic response above $T_{C}$. 
$\mathrm{cm}^{-3}$, estimated using the lattice constant $4.974 \AA$ ), we find that most of the $7 \mu_{B} \mathrm{Gd}^{3+}$ ions participate in the ferromagnetic transition at $70 \mathrm{~K}$ for sample $\mathrm{H}$.

In contrast, sample $\mathrm{M}$ shows a relatively weaker ferromagnetic transition at $70 \mathrm{~K}$ followed by a stronger transition near $45 \mathrm{~K}$. Furthermore, sample L shows a even weaker ferromagnetic transition at $70 \mathrm{~K}$, with a more abrupt transition near $45 \mathrm{~K}$. So the Mg-doped sample M (and L) shows weak (and weaker) ferromagnetic response between 45 and $70 \mathrm{~K}$, and most of the magnetic atoms order near $45 \mathrm{~K}$, unlike sample H. Similar to Figure (3.6), there is an interplay between free carriers and ferromagnetic behaviour. This matter will be further discussed in the next section, along with the transport data.

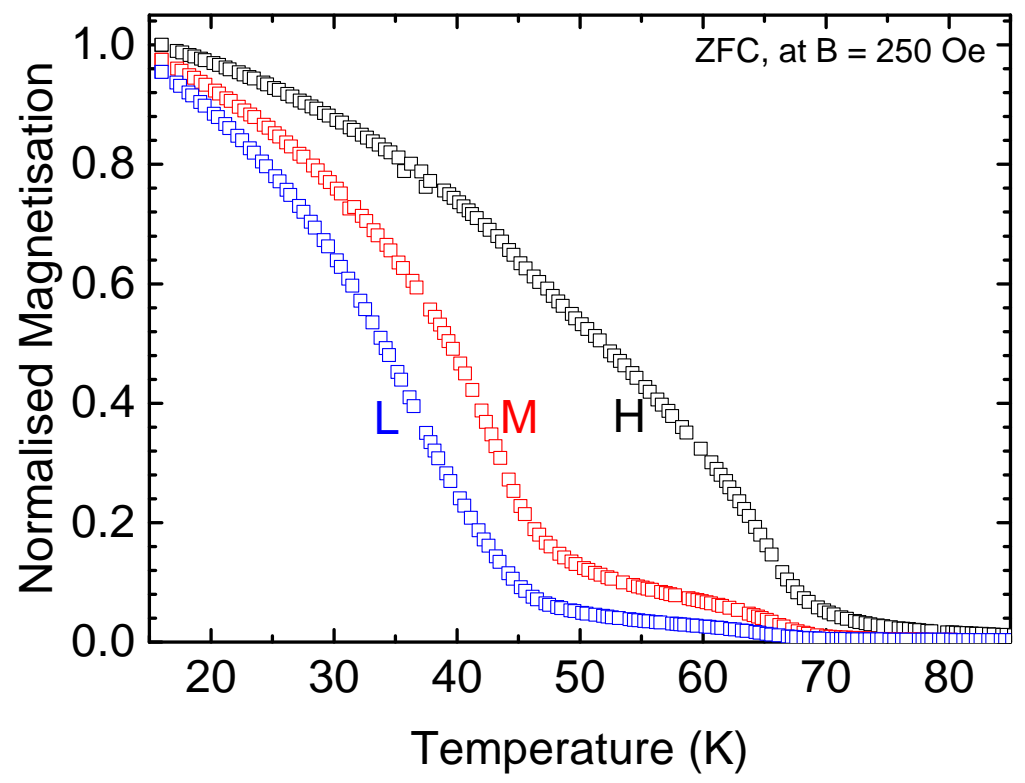

Figure 3.12: Temperature-dependent magnetisation of GdN films with different Mg-doping levels, zero field-cooled at 250 Oe. The magnetisation is normalised.

The ZFC magnetisation measurements on the same samples show ferromagnetic behaviours that are essentially the same as the FC measurements, as shown by Figure (3.12). This is because the applied field of 250 Oe is larger than the coercive fields of sample H, M and L, as observed in Figure (3.9). Since the samples are fully magnetised at low temperatures, the ZFC measurements do not deviate from the FC measurements. 

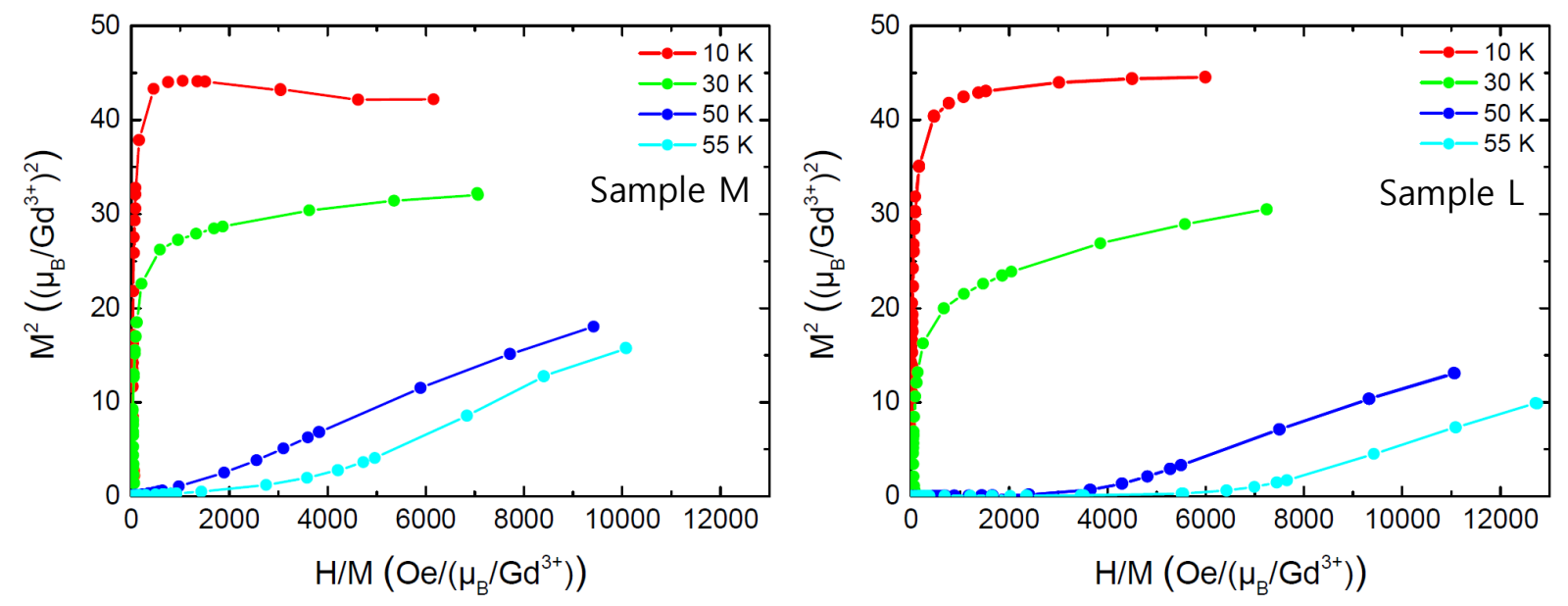

Figure 3.13: Arrott plots of sample M and L at various temperatures.

An Arrott plot is a plot of $M^{2}$ vs. $H / M$ for a material and they have been used by other groups in the REN community to estimate the value of $T_{C} \cdot{ }^{39}$ According to Ginzburg-Landau theory, the free energy $(F)$ of a ferromagnetic material near $T_{C}$ is given by:

$$
F(M)=-H M+a\left(T-T_{C}\right) M^{2}+b M^{4}+\ldots
$$

where $H$ is the applied field, $M$ is its magnetisation, and $a, b$ are arbitrary constants. $F=0$ when the ferromagnetic transition occurs, thus we have:

$$
M^{2}=\frac{1}{b} \frac{H}{M}-\frac{a}{b}\left(\frac{T-T_{C}}{T_{C}}\right)
$$

So given a plot of $M^{2}$ vs. $H / M$ at various temperatures, the straight line that goes through the origin without a y-intercept gives the value of $T_{C}$.

Figure (3.13) clearly shows that ferromagnetic transitions occur near $50 \mathrm{~K}$ for both sample $\mathrm{M}$ and $\mathrm{L}$, but with some contrasts. For sample $\mathrm{M}$, a line at $50 \mathrm{~K}$ appears quite straight and it goes through the origin, thus its $T_{C}$ lies very close to $50 \mathrm{~K}$. In contrast, sample L does not show a straight line through the origin, but we can easily estimate it to be somewhere between the curves at 30 and $50 \mathrm{~K}$. Thus sample $\mathrm{L}$ has its $T_{C}$ lower than sample M, which is consistent with the FC measurements in Figure (3.10). 


\subsection{Polaron Scenario}

To explain the link between charge carriers and magnetic exchange, Natali et al. have proposed a scenario involving magnetic polarons for GdN. ${ }^{18}$ According to calculations by Punya et al. ${ }^{21}$ a nitrogen vacancy $\left(V_{N}\right)$ in GdN provides up to three electrons, where two of them are bound to the $V_{N}$ in a singlet state, and the third electron is bound very weakly to the $V_{N}$, acting as a free carrier in the conduction band at high temperatures. These carriers are the main source of high conductivity in undoped GdN. If the temperature is decreased, then the carrier from $V_{N}$ starts to freeze out from the conduction band and occupy the third $V_{N}$ level. This causes the $V_{N}$ to have a magnetic moment of $1 \mu_{B}$ and also results in a large electron cloud forming around the $V_{N}$. Consequently, neighbouring $\mathrm{Gd}^{3+}$ ions can have their exchange mediated within the electron cloud, thus we have a magnetic polaron. ${ }^{40}$
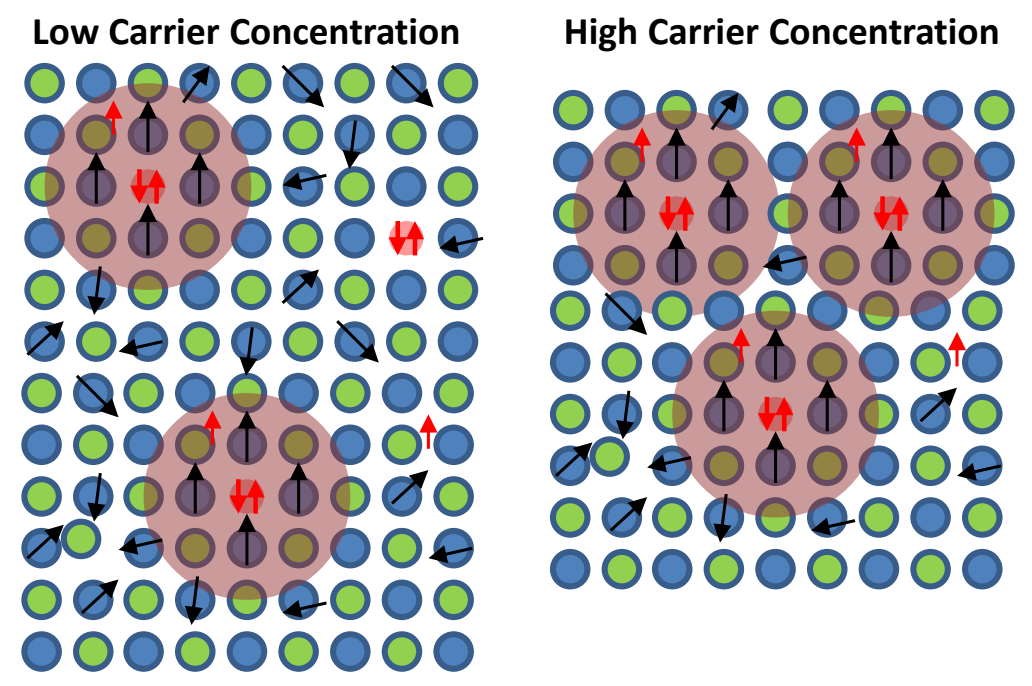

Figure 3.14: A diagram illustrating the magnetic ordering by polarons, for low and high carrier concentrations.

Figure (3.14) allows easier visualisation of the polaron scenario. The blue and green circles are $\mathrm{Gd}^{3+}$ and $\mathrm{N}^{3-}$ ions respectively, and the small red circles are $V_{N}$. The black arrows are the spins of $\mathrm{Gd}^{3+}$, and the red arrows are the spins of the electrons donated by $V_{N}$. Magnetic polarons are believed to form at $70 \mathrm{~K}$, and these are indicated by the large brown circles around $V_{N}$. We see that only the spins of $\mathrm{Gd}^{3+}$ ions near $V_{N}$ order at $70 \mathrm{~K}$, and the spins of $\mathrm{Gd}^{3+}$ ions outside the electron clouds are still randomly orientated. At high carrier concentration, i.e. high $V_{N}$ density, the polaron density is 
high enough such the electron clouds eventually overlap with each other. So when polarons nucleate, most of $\mathrm{Gd}^{3+}$ spins order, resulting in a uniform ferromagnetic phase. However, at low carrier concentration, the polaron density is low, thus the electron clouds do not overlap with each other. When the polarons nucleate, only the spins of $\mathrm{Gd}^{3+}$ ions near $V_{N}$ would order, and the rest of the $\mathrm{Gd}^{3+}$ ions in the film would not contribute to ferromagnetism until the intrinsic critical temperature of $\mathrm{Gd}$ is reached.

Based on this model, we propose to explain the ferromagnetic behaviour observed in Figure (3.10) in the following way. It is believed that magnetic polarons nucleate near $70 \mathrm{~K}$ in $\mathrm{GdN}$. So sample $\mathrm{H}$ goes through a ferromagnetic transition only once at $\sim 70 \mathrm{~K}$ because its carrier concentration is high enough for the polarons to strongly overlap, and all the $\mathrm{Gd}^{3+}$ spins order when polarons nucleate. As the Mg-doping level increases, the carrier concentration in GdN film is decreased, thus there is less overlapping between the polarons, as we saw in Figure (3.14). Therefore sample M (and L) shows weak (and weaker) ferromagnetic response between 45 and $70 \mathrm{~K}$ compared to sample $\mathrm{H}$ because there are less $\mathrm{Gd}^{3+}$ spins ordering at $\sim 70$ $\mathrm{K}$. Subsequently, more $\mathrm{Gd}^{3+}$ spins order at $\sim 45 \mathrm{~K}$ with decreasing carrier concentration, hence we see the strongest ferromagnetic response at $\sim 45 \mathrm{~K}$ for sample L. In fact, Figure (3.10) shows that most of the ferromagnetic response occurs near $45 \mathrm{~K}$ for sample $\mathrm{L}$, so its $T_{C}$ is effectively at $\sim 45 \mathrm{~K}$, which is in closer agreement with theoretical prediction for stoichiometric GdN. ${ }^{41,42}$

Returning to Figure (3.6), the observed shift in the resistivity anomalies is consistent with the proposed explanation for the shift in $T_{C}$. More resistive samples have less carriers to mediate exchange, so more $\mathrm{Gd}^{3+}$ spins order near $50 \mathrm{~K}$ instead of $70 \mathrm{~K}$. This means magnetic disorder scattering becomes stronger near $50 \mathrm{~K}$, thus the peaks show a tendency to move more towards $50 \mathrm{~K}$.

Overall, the observed shifts in resistivity anomalies and $T_{C}$ are consistent with the proposed magnetic polaron scenario. Natali et al. had already obtained similar results with epitaxial GdN films whose carrier concentrations were varied on a much smaller range, from $\sim 10^{18} \mathrm{~cm}^{-3}$ to $\sim 2 \times 10^{21} \mathrm{~cm}^{-3}$, by using different growth temperatures to tune carefully the $V_{N}$ concentration. ${ }^{18}$ Therefore we can confirm that the observed behaviour is likely due to the variation in density of carriers from $V_{N}$, and not directly related to the $\mathrm{Mg}$ dopants themselves. 


\section{Chapter 4}

\section{Photoconductivity}

Photoconductivity measurements were carried out on Mg-doped epitaxial GdN thin films to explore the effects of photo-excitation on the carrier concentration. Photoconductivity is observed when a photon is incident on the surface of a semiconductor and the photon energy is high enough to excite an electron from an occupied state to an unoccupied state. This results in both the excited electron and the hole left behind contributing towards the conductivity of the semiconductor. For a semiconductor with impurities, there can be localised impurity levels between the conduction band and the valence band, which can be occupied/unoccupied by the photo-generated electrons and holes. In particular, occupied states below the Fermi level may act as hole traps, and unoccupied states above the Fermi level may act as electron traps. These traps could prevent some of the photo-excited carriers from residing in the extended states, or permanently trap the carriers and form recombination centres. Such effects could be investigated through photoconductivity measurements to acquire a better understanding of the impurity levels in a semiconductor.

\subsection{Photoconductivity Characterisation Tech- niques}

Photoconductivity measurements were carried out in essentially the same manner as the measurement of resistivity as mentioned in Chapter 3. The only difference was that while the resistivity was being measured, there was light from a He-Ne laser (wavelength $633 \mathrm{~nm} \Longleftrightarrow$ photon energy $1.96 \mathrm{eV}$ ) 
of power $\sim 2.3 \mathrm{~mW}$ illuminating the sample. The optical gap of $\mathrm{GdN}$ is $\sim 1.3 \mathrm{eV}$ at room temperature, and it is reduced to $\sim 0.9 \mathrm{eV}$ below $T_{C},{ }^{43}$ so the energy of photons emitted from the He-Ne laser is large enough to stimulate photo-excitation in GdN. GaN has a band gap of $3.4 \mathrm{eV}$ so we do not expect any absorption by the capping layer. ${ }^{44}$ GaN capped GdN film has $\sim 15 \%$ reflectance at $633 \mathrm{~nm},{ }^{12}$ and the absorption coefficient of GdN at $633 \mathrm{~nm}$ is $\sim 7 \times 10^{4} \mathrm{~cm}^{-1}$ which gives $\sim 60 \%$ absorption for $\sim 130 \mathrm{~nm}$ thick GdN films. ${ }^{43}$ So overall, we expect about $\sim 50 \%$ of the incident photons to be absorbed by the GdN layer. Temperature-dependent measurement was carried out after the sample was under illumination for a sufficient amount of time, so that its photoconductivity reached steady-state.

The conductivity measured under illumination is the sum of the conductivity in dark and the photoconductivity $(\Delta \sigma)$ due to the photo-generated carriers

$$
\Delta \sigma=e\left(\mu_{n} \delta n+\mu_{p} \delta p\right)
$$

where $\mu_{n}$ and $\mu_{p}$ are electron and hole mobilities, respectively, and $\delta n$ and $\delta p$ are excess electron and hole concentrations due to photo-excitation, respectively. Thus the photoconductivity is obtained by taking the difference between the conductivity under illumination and in dark.

Time-dependent measurement of photoconductivity was carried out by switching the laser off after the steady-state was established, and measuring the conductivity as a function of time at a given temperature. For heavily $\mathrm{Mg}$ doped GdN films, the decay in conductivity after the switching off was too rapid $\left(<10^{-1} s\right)$ to be meaningfully analysed in the time domain. Thus frequency-dependent measurements were conducted to analyse the decay in the frequency domain. This was carried out in essentially the same way as the time-dependent measurement, but a mechanical chopper was placed in front the laser and a lock-in amplifier was used to pick up the signal. The chopper and the lock-in amplifier allowed the photoconductivity to be measured for frequency values ranging from $5 \mathrm{~Hz}$ up to $20 \mathrm{kHz}$. 


\subsection{Temperature-dependent Photoconductiv- ity}

The temperature-, time- and frequency-dependent photoconductivity measurements were carried out on Mg-doped epitaxial GdN thin films shown in Figure (4.1). Again, they were named according to their carrier concentration for convenience, where VL stands for "very low". Note that not all the measurements were carried out on each sample, due to practical reasons such as the change in conductivity being too small, the photoconductive response being too fast or too slow etc.

\begin{tabular}{|l|l|l|l|}
\hline Name & Sample & $\begin{array}{l}\text { Mg Flux } \\
\text { (Torr) }\end{array}$ & $\begin{array}{l}\text { Carrier Conc. }\left(\mathrm{cm}^{-3}\right) \\
\text { At Room Temp. }\end{array}$ \\
\hline H & SCO987 & 0 & $\sim 2 \times 10^{20}$ \\
\hline M & SCO981 & $1.28 \times 10^{-10}$ & $\sim 6 \times 10^{18}$ \\
\hline L & SCO983 & $2.09 \times 10^{-10}$ & $\sim 5 \times 10^{17}$ \\
\hline VL & SCO988 & $5.94 \times 10^{-10}$ & $\sim 1 \times 10^{17}$ \\
\hline
\end{tabular}

Figure 4.1: Samples used for photoconductivity measurements.

Figure (4.2) shows the temperature-dependent resistivity measured in dark and under illumination, for sample L, M and $\mathrm{H}$. All samples show reduced resistivity under illumination for all measured temperatures, demonstrating photoconductive responses. The temperature-dependent resistivity in dark was discussed in Chapter 3, and we see that the behaviour under illumination also follows the behaviour in dark, but with a subtle change. Notice how the TCR above $150 \mathrm{~K}$ and below $10 \mathrm{~K}$ becomes slightly less negative when the samples are illuminated, which is quite apparent for sample $\mathrm{H}$ and L. Furthermore, the resistivity peaks shift to higher temperature, which is especially apparent for sample M. Therefore, under illumination, the temperature-dependent resistivity curves behave as if they had more carriers, clearly demonstrating the increase in carrier concentration due to photo-excitation in these samples. 


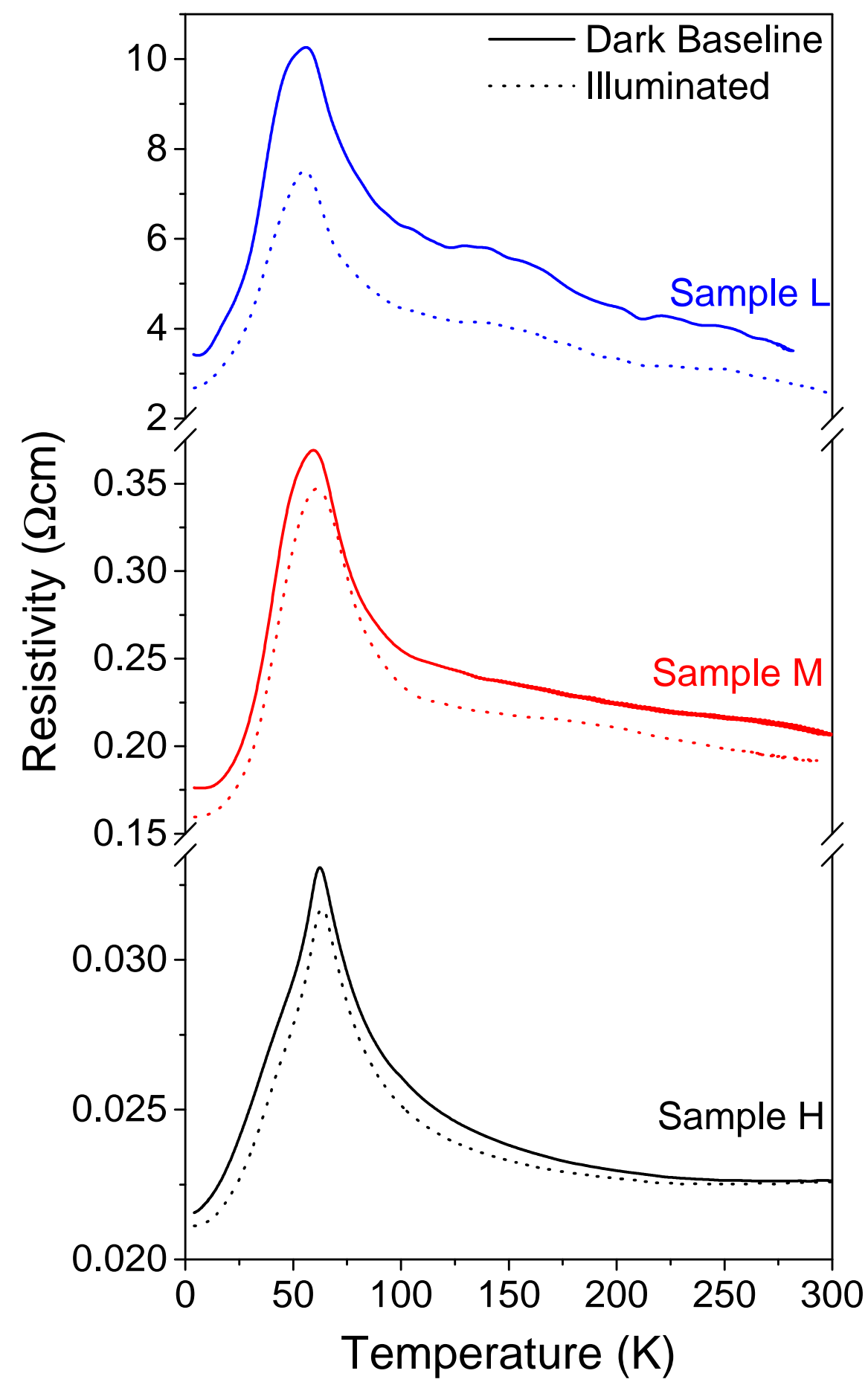

Figure 4.2: Temperature-dependent resistivity of Mg-doped GdN films when illuminated under $633 \mathrm{~nm}$ He-Ne laser, along with the resistivity measured in dark. The resistivity under illumination was measured after steady-state was established. 
The photoconductivity in n-type GdN can be understood in terms of competition between different transitions as illustrated in Figure (4.3). The $V_{N}$ in GdN forms a tail of localised impurity states below the conduction band mobility edge, and it has been reported that these act as electron traps. $^{27}$ Deep-level hole traps act as recombination centres because when an electron is photo-excited, it leaves a hole behind which is quickly captured by the hole trap. So when the GdN film is under illumination, there are electrons photo-excited from the valence band to the conduction band $(G)$, holes forming recombination centres $(H)$, free electrons being trapped by the $V_{N}(X)$, trapped electrons detrapping via thermal excitation $(D)$, and free electrons recombining $(R)$. Due to spatial separation, direct recombinations between the electron trap and hole trap are forbidden. While illumination $(G)$ results in excess electrons (density given by $\delta n=\delta n_{c}+\delta n_{t}$ ), only the excess free carriers (density $\delta n_{c}$ ) in the conduction band actually contribute to the increase in conductivity, and the excess trapped carriers (density $\delta n_{t}$ ) serve only as a pool of potential free carriers. Thus the photoconductivity is proportional to the density of excess free carriers $\delta n_{c}$, not $\delta n$. When steady-state is reached, regardless of the value of $G$, an equilibrium is established between the conduction band and the electron traps such that $D=X$. Therefore the distribution of carriers between the extended states and localised states is described by a quasi-Fermi level that lies somewhere between the conduction band and the electron traps.

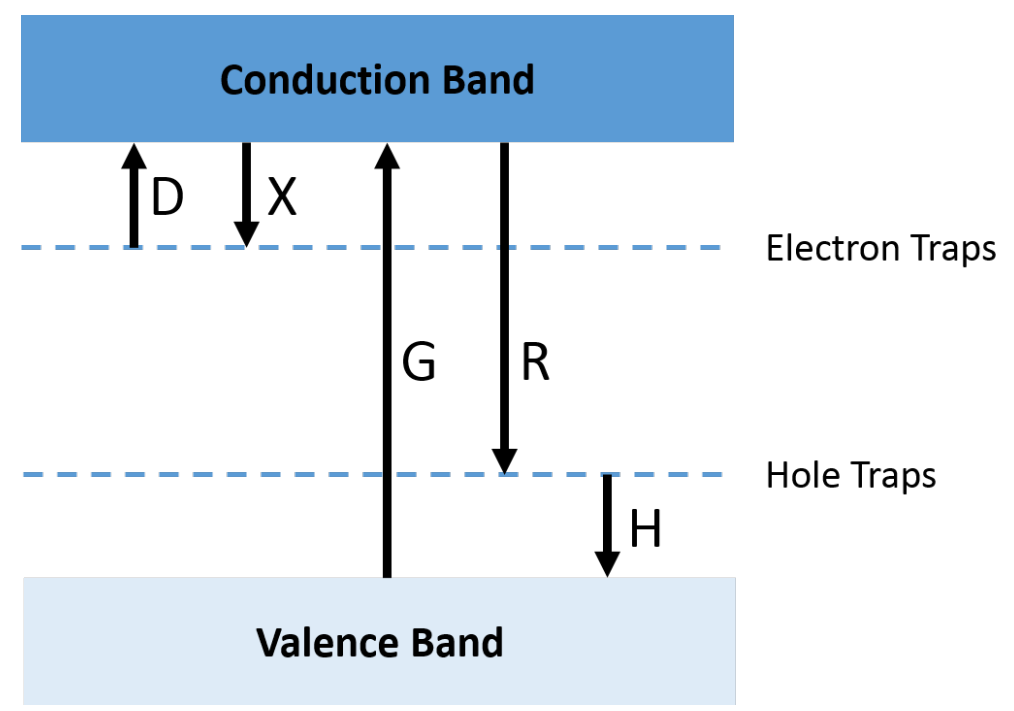

Figure 4.3: Allowed transitions for semiconductor with electron and hole traps. $X$ : trapping of electrons, $D$ : de-trapping of electrons, $G$ : photo-excitation of electrons under illumination, $R$ : recombination of electrons with trapped holes, $H$ : capturing of holes. 


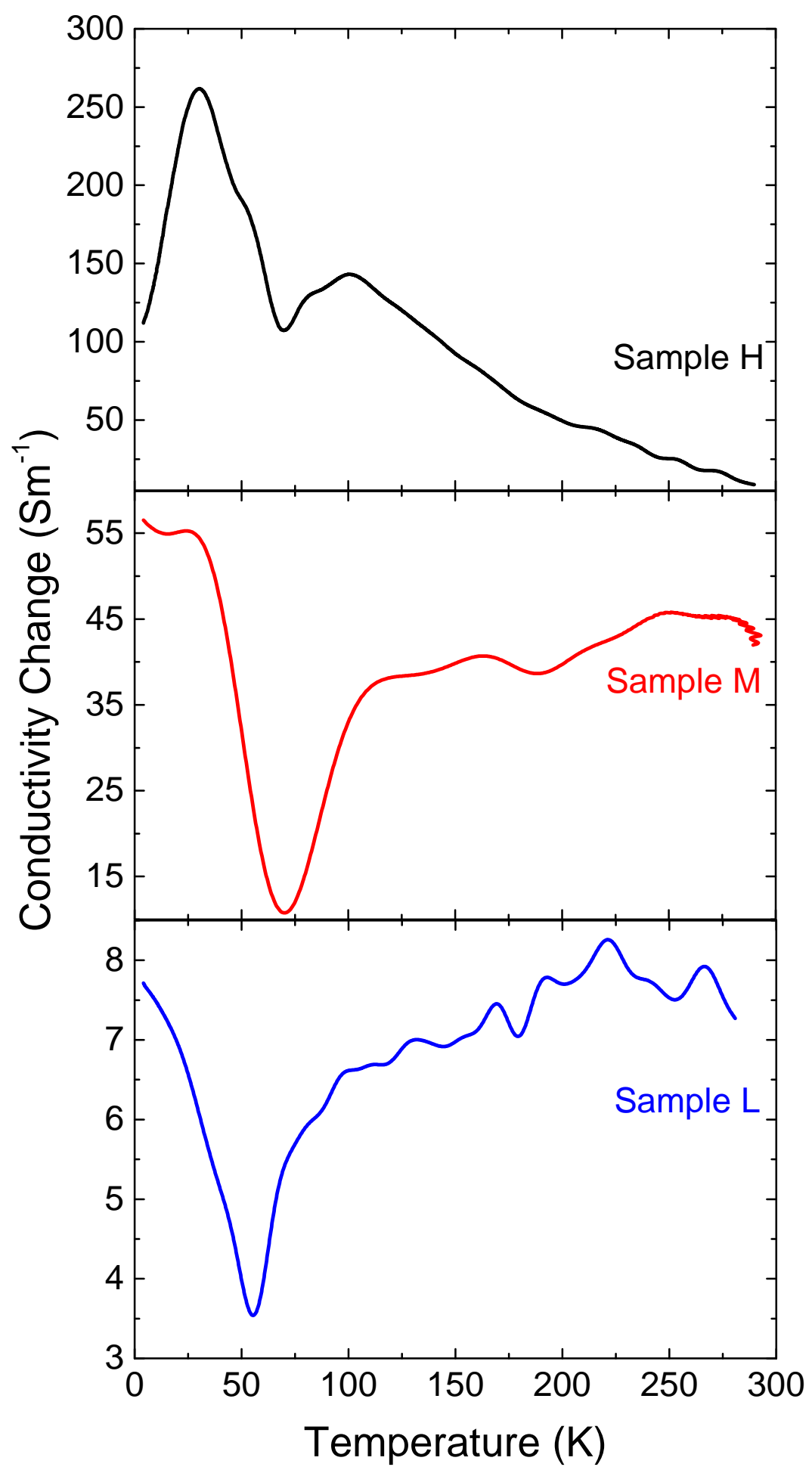

Figure 4.4: Temperature-dependent photoconductivity of Mg-doped GdN films at steady-state. Note that the data is not plotted on the same graph, it is just a stack of 3 separate graphs. This is due to sample $\mathrm{M}$ and $\mathrm{H}$ having an overlapping range of photoconductivity. 
Figure (4.4) shows the difference in conductivity measured in dark and under illumination as a function of temperature, i.e. temperature-dependent photoconductivity for sample L, M and H. Sample L shows decreasing photoconductivity with decreasing temperature, from $\sim 200 \mathrm{~K}$ down to $T_{C}$. This behaviour can be explained by interpreting photoconductivity as a result of a rise in the quasi-Fermi level. ${ }^{45}$ When the sample is illuminated, the quasi-Fermi level rises towards the conduction band, adding $\delta n_{c}$ to the conduction band and $\delta n_{t}$ to the trap level. Now, sample $L$ is heavily doped with $\mathrm{Mg}$, having a relatively low carrier concentration of $\sim 5 \times 10^{17} \mathrm{~cm}^{-3}$, which implies that its quasi-Fermi level is located far below the conduction band. The Fermi function becomes sharper at lower temperature, so when the quasi-Fermi level rises due to illumination, more $\delta n_{t}$ are introduced for a given amount of introduced $\delta n_{c} \cdot{ }^{27}$ Since the density of recombination centres $\rho$ is equal to the total density of excess carriers, $\rho=\delta n=\delta n_{c}+\delta n_{t}$, there is higher $\rho$ per $n_{c}$ at lower temperature, implying more recombined electrons when the steady-state is reached. Therefore sample L shows less photoconductivity at lower temperature. At temperatures higher than 200 $\mathrm{K}$, the Fermi function is broad enough such that the balance between $\delta n_{c}$ and $\delta n_{t}$ is effectively unchanged when illuminated, so the photoconductivity shows little temperature-dependence. Below $T_{C}$, the photoconductivity shows an increasing trend because the sample undergoes exchange splitting of the conduction band, moving the conduction band closer to the quasi-Fermi level. Thus the ratio $\delta n_{c} / \delta n_{t}$ increases when the temperature decreases, resulting in the observed trend.

Sample M also shows behaviour similar to that of sample L, but with a slight difference. From room temperature down to $T_{C}$, the photoconductivity decreases but it is not as strong as observed in sample L. This is because sample $\mathrm{M}$ has carrier concentration of $\sim 6 \times 10^{18} \mathrm{~cm}^{-3}$, which is higher than sample L. So its quasi-Fermi level is located somewhat higher, such that a good balance between $\delta n_{c}$ and $\delta n_{t}$ is still maintained even at lower temperatures. Below $T_{C}$, the photoconductivity shows an increasing trend just like sample L. Below $\sim 30 \mathrm{~K}$, the Fermi function eventually becomes too sharp such that the increasing trend stops and the behaviour observed near room temperature is repeated.

In contrast to sample $\mathrm{L}$ and $\mathrm{M}$, sample $\mathrm{H}$ shows increasing photoconductivity with decreasing temperature. Following from the explanation above, this is because sample $H$ has a high carrier concentration of $\sim 2 \times 10^{20}$ $\mathrm{cm}^{-3}$. Its quasi-Fermi level is likely to be located somewhere very close to the conduction band, much higher than its trap levels. The Fermi function 
becomes sharper at lower temperature, but because this sharpening still results in greater occupancy of $n_{c}$ than $n_{t}$, the ratio $\delta n_{c} / \delta n_{t}$ increases with decreasing temperature. When the Fermi function becomes sharp enough near $\sim 100 \mathrm{~K}$, the photoconductivity eventually shows the decreasing trend like the other samples until $T_{C}$. Below $T_{C}$ the photoconductivity increases like the other samples, but below $\sim 30 \mathrm{~K}$, a decreasing trend is observed which suggests that the Fermi function becomes sharp enough to repeat the behaviour observed just above $T_{C}$.

Note that the magnitude of photoconductivity is not constant throughout the samples. The average change in conductivity is in the order of $\sim 100$, $\sim 35, \sim 6 \mathrm{Sm}^{-1}$ for sample $\mathrm{H}, \mathrm{M}$ and L, respectively, so we see greater conductivity changes in samples with more carriers. This matter will be further discussed after the discussion of persistent photoconductivity.

\subsection{Persistent Photoconductivity}

Suppose a photoconductive sample has been illuminated until steady-state is reached, and the light is switched off afterwards. Persistent photoconductivity (PPC) refers to the phenomenon where photoconductivity is still observed even when the sample is not under illumination any more, and it can give ideas about the dynamics of photo-generated carriers in the sample. PPC can last for a very long time, even days after the switching off of the light. The origin of PPC varies from material to material, and in GdN it has been attributed to the deep states formed by nitrogen vacancies $\left(V_{N}\right) \cdot{ }^{27}$

PPC was observed for sample $\mathrm{H}$ and $\mathrm{M}$ at $100 \mathrm{~K}$, as shown by the timedependent photoconductivity measurements in Figure (4.5). The measurements were carried out at $100 \mathrm{~K}$ because the decay at higher temperature was too fast to be analysed properly. Right after the light is turned off, the photoconductivity decays rapidly in a non-exponential manner, as indicated by the initial non-linear drop. However, when the photoconductivity has decreased to a sufficiently small value, it starts to follow an exponential decay, which is indicated by the linear drop. The initial non-exponential decrease of photoconductivity may be explained by considering the allowed transitions shown in Figure (4.3). At steady-state, $D=X$ and $G=R$ such that

$$
\frac{d}{d t}\left(\delta n_{c}\right)=G-R+D-X=0, \quad \frac{d}{d t}\left(\delta n_{t}\right)=X-D=0
$$



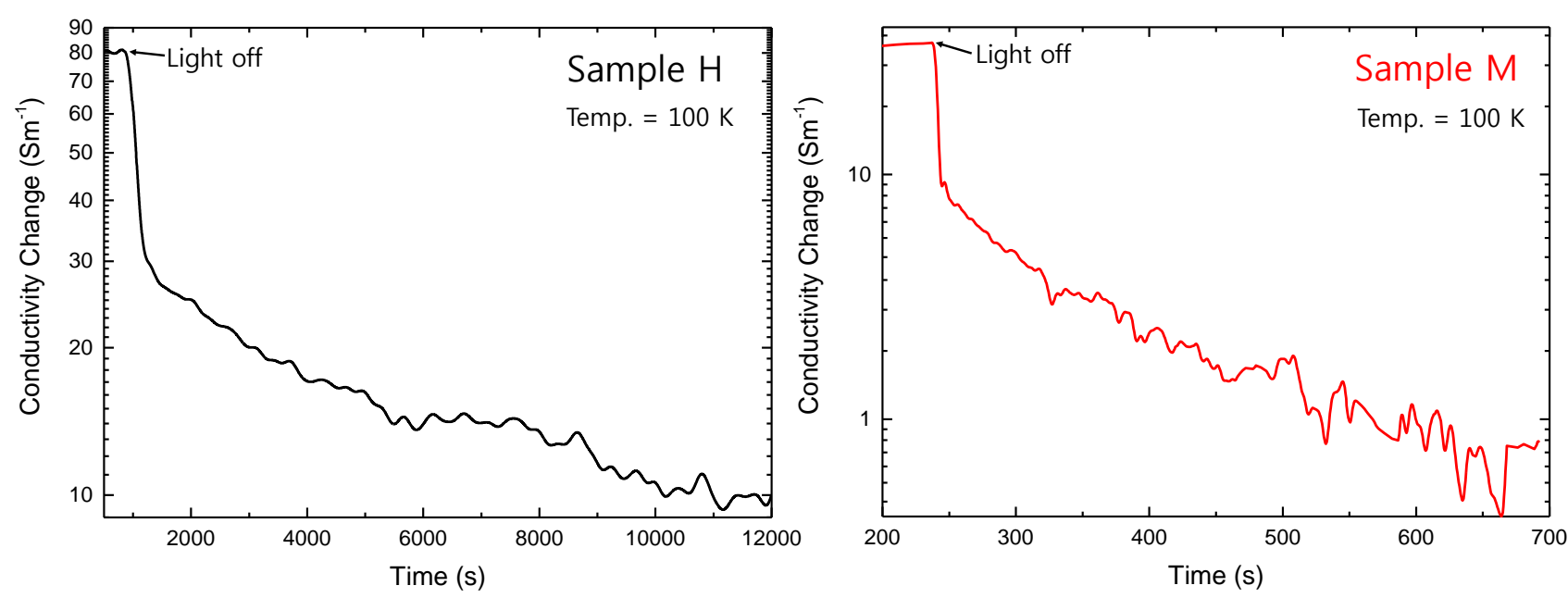

Figure 4.5: Photoconductivity as a function of time for sample $\mathrm{H}$ and M, at 100 $\mathrm{K}$. The light was turned off after the steady-state was reached by the samples.

Immediately after the light is switched off, $G$ suddenly becomes 0 so that $R>\rightarrow$, whereas $D=X$ is still maintained for a short time since it needs a finite amount of time to respond to the change in $\delta n_{c}$. So we expect a burst of depopulating excess free carriers at a rate $d\left(\delta n_{c}\right) / d t=-R$ and consequently a rapid drop in the photoconductivity, until the excess carriers in the trap levels respond to the decrease in $\delta n_{c}$ such that $D>X$ and start populating the conduction band. At this point, $d\left(\delta n_{c}\right) / d t=$ $-R+D-X$ is lowered in magnitude because $D-X>0$, and the decay in photoconductivity is slowed down.

An argument by Warring et al. explains the exponential decay after the nonexponential decay. ${ }^{27}$ The density of excess carriers $\left(\delta n_{c}, \delta n_{t}\right)$ are determined by the quasi-Fermi level $\left(E_{F}^{*}\right)$, whose first-order approximations near the Fermi level $\left(E_{F}\right)$ are given by

$$
\begin{gathered}
\delta n_{c}=n_{c}\left(E_{F}^{*}-E_{F}\right) / k_{B} T \\
\delta n_{t}=D\left(E_{F}\right)\left(E_{F}^{*}-E_{F}\right)
\end{gathered}
$$

where $D\left(E_{F}\right)$ is the density of localised states at $E_{F}$. Since $\rho=\delta n_{c}+\delta n_{t}$, combining above expressions lead to

$$
\delta n_{c}=\frac{\rho}{1+D\left(E_{F}\right) k_{B} T / n_{c}}
$$

which shows that $\delta n_{c} \propto \rho$ at a given temperature. Note that the recombination centres $\rho$ are filled at the recombination rate $(R)$, given by $R=-\Gamma n_{c} \rho$ 
where $\Gamma$ is some constant. ${ }^{46}$ Therefore we have

$$
\frac{d \rho}{d t} \propto R=-\Gamma n_{c} \rho
$$

When $n_{c}$ becomes closer enough to its dark value, it has smaller timedependence, thus $\frac{d \rho}{d t} \propto \rho$ and $\rho$ decays in an exponential manner. It subsequently follows that $\delta n_{c}$ also decays exponentially, since $\delta n_{c} \propto \rho$. The slope of the linear trend gives the characteristic time $(\tau)$ of the exponential decay $\exp (-t / \tau)$ and we obtain $\tau$ of $\sim 13000 \mathrm{~s}$ and $\sim 170 \mathrm{~s}$ for sample $\mathrm{H}$ and $\mathrm{M}$, respectively. The characteristic time $(\tau)$ is dramatically decreased with a moderate level of Mg-doping, almost by 2 orders of magnitude.

It is worth pointing out that PPC has been observed in group-III nitride thin films. In particular, they have been observed in various p-type GaN films doped with Mg, grown by methods such as MOCVD and MBE. ${ }^{47,48,49,50}$ It has been suggested that the PPC effects in p-type GaN originate from the hole traps provided by $\mathrm{Mg}$ impurities. ${ }^{49}$ Thus we suspect that $\mathrm{Mg}$-doping provides more hole traps that act as recombination centres in n-type GdN, consequently leading to faster PPC decays in GdN films with higher $\mathrm{Mg}$ doping levels.
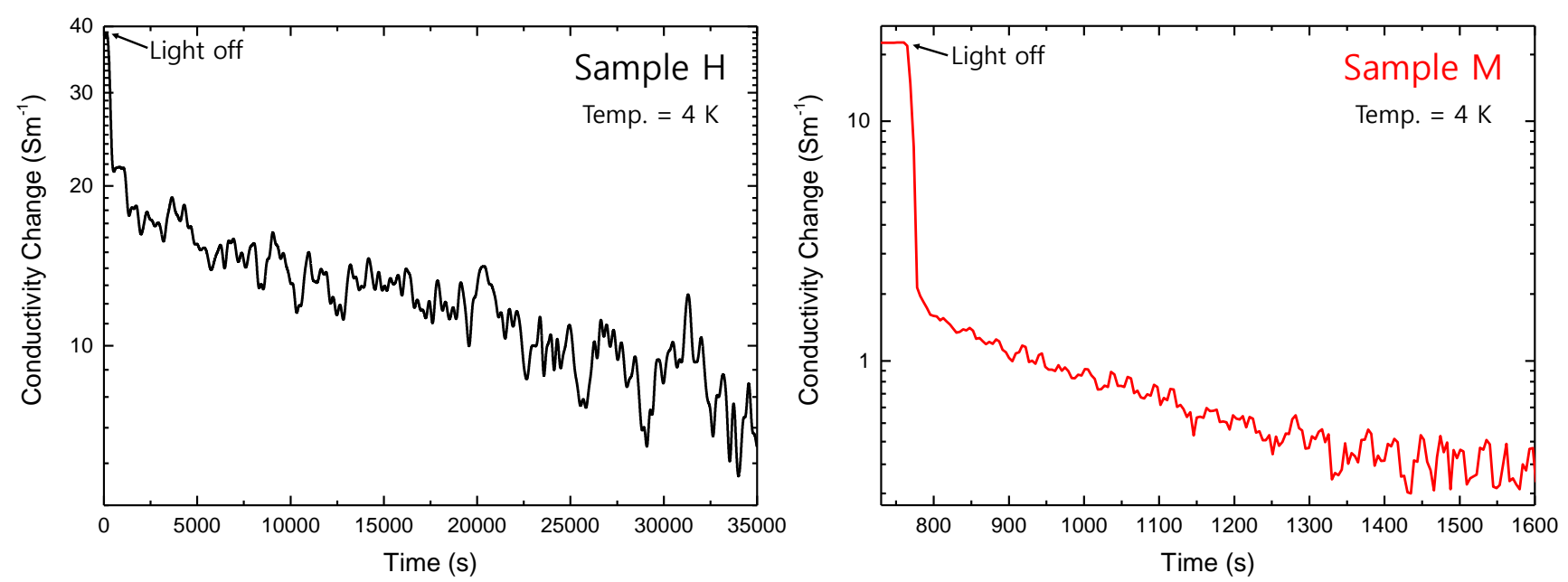

Figure 4.6: Photoconductivity as a function of time for sample $\mathrm{H}$ and $\mathrm{M}$, at $4 \mathrm{~K}$. The light was turned off after the steady-state was reached by the samples.

We see from Figure (4.6) that PPC behaviours at $4 \mathrm{~K}$ are consistent with the behaviours observed at $100 \mathrm{~K}$ for both sample $\mathrm{H}$ and $\mathrm{M}$. However, the characteristic times of the exponential decay are $\sim 45000 \mathrm{~s}$ and $\sim 600 \mathrm{~s}$ for sample $\mathrm{H}$ and $\mathrm{M}$, respectively, thus the decay is slowed down by a factor 
of $\sim 3$ compared to $100 \mathrm{~K}$. This is because the detrapping of electrons is a thermal process, thus it occurs less frequently at lower temperatures. Since the trapped electrons must be detrapped before recombination, we observe slower photoconductive decay at lower temperatures.

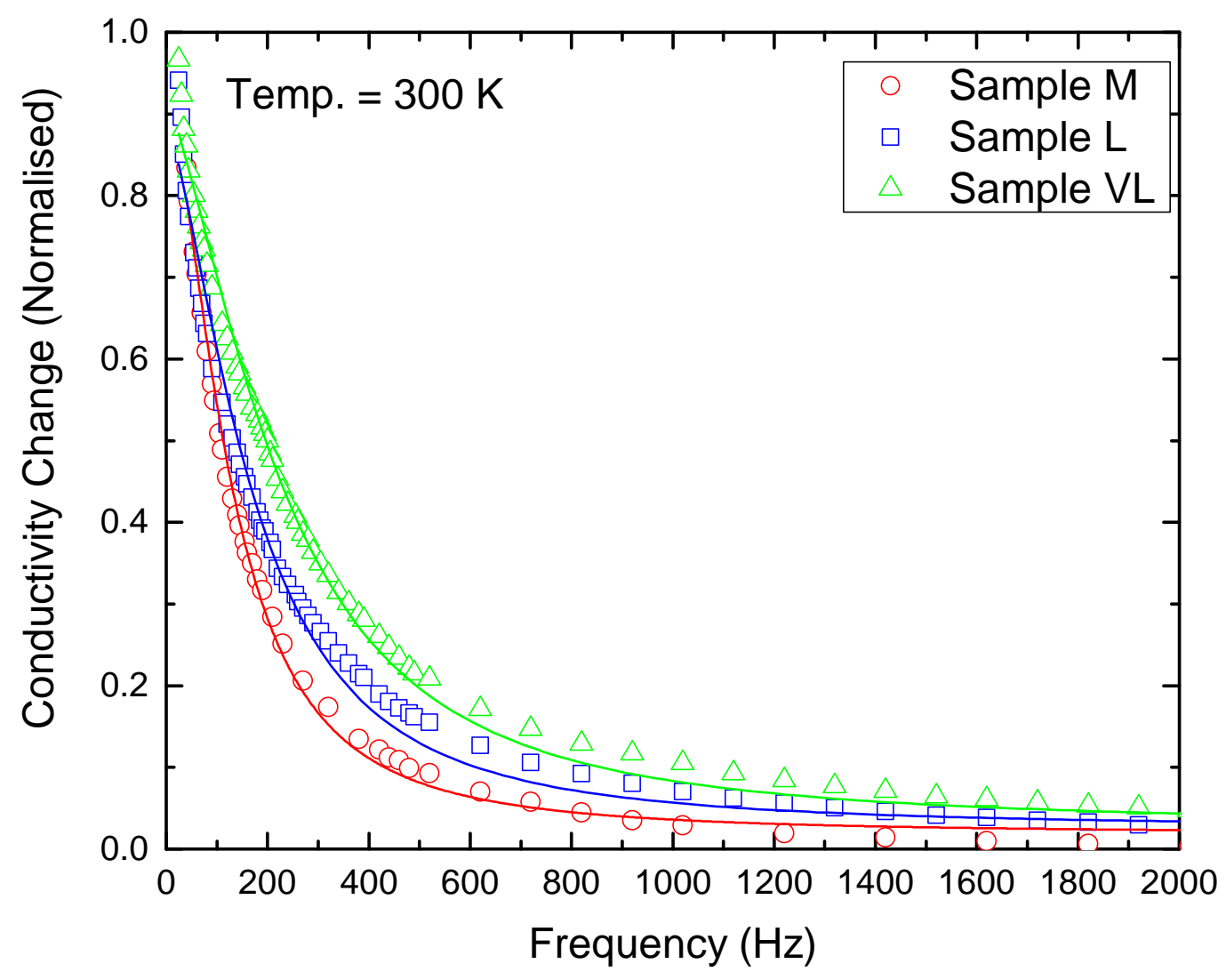

Figure 4.7: Normalised photoconductivity as a function of frequency, at room temperature. Lines through the data points are fitted Lorentzian functions.

The frequency-dependent photoconductivity measurements at room temperature also display a reduction in PPC with higher Mg-doping level, which is shown in Figure (4.7). The Fourier transform of an exponential decay $\exp (-a t)$ is a Lorentzian function in the form $\frac{2 a}{a^{2}+4 \pi^{2} f^{2}}$ where $f$ is the frequency. We saw in Figure (4.5) and (4.6) that decays of photoconductivity at $4 \mathrm{~K}$ and $100 \mathrm{~K}$ are not purely exponential, so Lorentzian functions are unlikely to fit the obtained frequency-dependent data perfectly. Still, 
the frequency-dependent decays persist longer for GdN films with higher Mg-doping levels, clearly indicating faster responses in the time domain for these samples. To roughly estimate the value of $\tau(=1 / a)$, a Lorentzian function was fitted for each curve. We find that $\tau$ is $\sim 1.2 \mathrm{~ms}, \sim 0.8 \mathrm{~ms}, \sim 0.6$ ms for sample M, L and VL, respectively. Although the decrease in $\tau$ is not as dramatic as we saw in the time-dependent measurement at low temperatures, the samples with higher Mg-doping levels certainly show reduced decay times in a systematic manner.

Returning to Figure (4.4), now it is possible to explain why samples with higher Mg-doping levels displayed smaller steady-state photoconductivity. The amount of excess free electrons $\left(\delta n_{c}\right)$ is determined by the competition between the allowed transitions. Steady-state is reached when there is just enough $\delta n_{c}$ such that electrons are continuously generated by photoexcitation and annihilated at the same rate through electron-hole recombination $(G=R)$, and the trapping and detrapping rates are equal $(D=X)$. Since the samples with higher Mg-doping levels show higher recombination rate, these samples need less $\delta n_{c}$ to reach steady-state. Therefore the average magnitude of steady-state photoconductivity decreases with higher Mg-doping level.

In summary, undoped and Mg-doped epitaxial GdN thin films all showed clear photoconductive responses at temperatures between $4 \sim 300 \mathrm{~K}$. PPC was also observed for all of them, supporting the presence of electron traps associated with $V_{N}$ levels in GdN films. Both the magnitude of photoconductivity and the decay time of PPC were observed to decrease with increasing Mg-doping level, which suggests $\mathrm{Mg}$ impurities providing more recombination centres in Mg-doped GdN films. 


\section{Chapter 5}

\section{Conclusion}

To summarise, Mg-doped epitaxial GdN thin films with varying Mg-doping levels were grown on [0001]-orientated wurtzite AlN buffer layer using MBE, and their structural, electric, magnetic and photoconductive properties were investigated.

The GdN layers were grown at $650{ }^{\circ} \mathrm{C}$ for epitaxial growth, and their epitaxial character was confirmed by in situ RHEED observations. Characterisation through XRD showed that the grown films are [111]-orientated, as favoured by the hexagonal face of AlN buffer layer. Overall, there was no significant systematic variation in the crystallographic structure of the films with increasing level of Mg-doping, for Mg concentrations up to $\sim 5 \times 10^{19}$ atoms $/ \mathrm{cm}^{3}$. However, from $\mathrm{Mg}$ concentration of $\sim 2 \times 10^{20}$ atoms $/ \mathrm{cm}^{3}$, a clear deterioration in the crystalline quality was seen.

Hall effect measurement showed that an undoped GdN film has n-type carrier concentration of $7 \times 10^{20} \mathrm{~cm}^{-3}$ at room temperature, likely due to electron-doping by $V_{N}$. The carrier concentration was observed to decrease with increasing level of Mg-doping, down to $5 \times 10^{15} \mathrm{~cm}^{-3}$ for a film with $\mathrm{Mg}$ concentration of $5 \times 10^{19}$ atoms $/ \mathrm{cm}^{3}$. An increase in the room temperature resistivity of the films was also observed, from 0.002 to $600 \Omega \mathrm{cm}$ as the Mg-doping level increased, resulting in semi-insulating films for $\mathrm{Mg}$ concentrations up to $5 \times 10^{19}$ atoms $/ \mathrm{cm}^{3}$. Overall, electron compensation in $\mathrm{GdN}$ via Mg-doping is clearly demonstrated.

Temperature-dependent resistivity measurements showed a change from positive to negative TCR above $150 \mathrm{~K}$ as the Mg-doping level increased, approaching the behaviour expected from an intrinsic semiconductor. Re- 
sistivity anomalies were observed to peak at $\sim 70 \mathrm{~K}$ for an undoped film, and down to $\sim 55 \mathrm{~K}$ for films with lower carrier concentration.

The saturation magnetisation of the films were found to be close to $7 \mu_{B} / \mathrm{Gd}^{3+}$, with a small variation depending on the Mg-doping level. The saturation magnetisation of $6.2 \mu_{B} / \mathrm{Gd}^{3+}$ for an undoped film was increased up to $6.8 \mu_{B} / \mathrm{Gd}^{3+}$ for a heavily Mg-doped film, showing a slight tendency to become closer to $7 \mu_{B} / \mathrm{Gd}^{3+}$ as the carrier concentration decreases. The coercive field of $\sim 140$ Oe for an undoped film was reduced to $\sim 90$ Oe for the Mg-doped films, but the amount of reduction was independent of the Mg-doping level.

Temperature-dependent magnetisation measurements exhibited substantial variations between the films, with a Curie temperature of $\sim 70 \mathrm{~K}$ for an undoped film reduced down to $\sim 50 \mathrm{~K}$ for a heavily Mg-doped film. Such lowering of $T_{C}$ with decreasing carrier concentration, along with the shift in anomalies observed in the temperature-dependent resistivity measurement, is consistent with expectations from the magnetic polaron scenario proposed by Natali et al. ${ }^{18}$

All Mg-doped GdN films were found to be photoconductive with varying magnitudes and response times. The average magnitude of photoconductivity at steady-state was observed to decrease with increasing Mg-doping level, from $\sim 100 \mathrm{Sm}^{-1}$ for an undoped film to $\sim 6 \mathrm{Sm}^{-1}$ for a heavily $\mathrm{Mg}$ doped film. By interpreting photoconductivity as a rise in a quasi-Fermi level between the conduction band and the electron traps, the temperaturedependent photoconductive behaviours observed in the GdN films could be explained.

Time-dependent photoconductivity measurements revealed PPC in the GdN films, where a rapid non-exponential drop followed by a slower exponential decay is observed at $100 \mathrm{~K}$. The exponential decay time of $13000 \mathrm{~s}$ for an undoped film was reduced to $170 \mathrm{~s}$ with a moderate level of Mg-doping. Similar behaviours were observed at $4 \mathrm{~K}$, but the decay times were slowed down by a factor of $\sim 3$. Room temperature frequency-dependent measurements also displayed similar behaviour, showing photoconductive response times systematically decreasing with increasing level of Mg-doping. This raises the possibility of $\mathrm{Mg}$ impurities providing hole traps that act as recombination centres in n-type GdN films. Overall, the results point towards the presence of electron traps associated with $V_{N}$ levels in GdN films, and demonstrates that impurity levels in GdN can be probed through photoconductivity measurements. 


\section{Bibliography}

[1] F. Natali, B. J. Ruck, N. O. V. Plank, H. J. Trodahl, S. Granville, C. Meyer, and Walter R. L. Lambrecht. Rare-earth mononitrides. Prog. Mats. Sci., 58(132409), 2013.

[2] USGS. Rare Earth Elements - Critical Resources for High Technology. (http://pubs.usgs.gov/fs/2002/fs087-02/), 2005.

[3] F. Hulliger. Magnetic properties of the rare earth pnictides. J. Magn. Magn. Mater., 8(183), 1978.

[4] C.-G. Duan, R. F. Sabirianov, W. N. Mei, P. A. Dowben, S. S. Jaswal, and E. Y. Tsymbal. Electronic, magnetic and transport properties of rare-earth monopnictides. J. Phys.: Condens. Matter, 19(315220), 2007.

[5] P. Larson, W. R. L. Lambrecht, A. Chantis, and M. van Schilfgaarde. Electronic structure of rare-earth nitrides using the LSDA+U approach: Importance of allowing $4 \mathrm{f}$ orbitals to break the cubic crystal symmetry. Phys. Rev. B, 75(045114), 2007.

[6] F. Nasirpouri and A. Nogaret (eds.). Nanomagnetism and Spintronics: Fabrication, Materials, Characterization and Applications. World Scientific Publishing Company, Singapore, 2011.

[7] F. Natali, N.O.V. Plank, J. Galipaud, B. J. Ruck, H. J. Trodahl, F. Semond, S. Sorieul, and L. Hirsch. Epitaxial growth of GdN on silicon substrate using an AlN buffer layer. J. Cryst. Growth, 312(3583), 2010.

[8] K. Senapati, M. G. Blamire, and Z. H. Barber. Spin-filter josephson junctions. Nat. Mater., 10(849), 2011.

[9] M. A. Scarpulla, C. S. Gallinat, W. S. Mack, J. S. Speck, and A. C. Gossard. GdN (111) heteroepitaxy on GaN (0001) by $\mathrm{N}_{2}$ plasma and $\mathrm{NH}_{3}$ molecular beam epitaxy. J. Cryst. Growth, 311(1239), 2009. 
[10] H. Yoshitomi, S. Kitayama, T. Kita, O. Wada, M. Fujisawa, H. Ohta, and T. Sakurai. Optical and magnetic properties in epitaxial GdN thin films. Phys. Rev. B, 83(155202), 2011.

[11] F. Leuenberger, A. Parge, W. Felsch, K. Fauth, and M. Hessler. GdN thin films: Bulk and local electronic and magnetic properties. Phys. Rev. B, 72(014427), 2005.

[12] S. Granville, B. J. Ruck, F. Budde, A. Koo, D. J. Pringle, F. Kuchler, A. R. H. Preston, D. H. Housden, N. Lund, A. Bittar, G. V. M. Williams, and H. J. Trodahl. Semiconducting ground state of GdN thin films. Phys. Rev. B, 73(235335), 2006.

[13] C. Meyer, B. J. Ruck, J. Zhong, S. Granville, A. R. H. Preston, G. V. M. William, and H. J. Trodahl. Near-zero-moment ferromagnetism in the semiconductor SmN. Phys. Rev. B, 78(174406), 2008.

[14] G. Busch. Magnetic properties of rare-earth compounds. J. Appl. Phys., 38(1386), 1967.

[15] R. A. Cutler and A. W. Lawson. Synthesis and magnetic behavior of GdN. J. Appl. Phys., 46(2739), 1975.

[16] K. Khazen, H. J. von Bardeleben, J. L. Cantin, A. Bittar, S. Granville, H. J. Trodahl, and B.J. Ruck. Ferromagnetic resonance study of GdN thin films with bulk and extended lattice constants. Phys. Rev. B, 74(245330), 2006.

[17] B. M. Ludbrook, I. L. Farrell, M. Kuebel, B. J. Ruck, A. R. H. Preston, H. J. Trodahl, L. Ranno, R. J. Reeves, and S.M. Durbin. Growth and properties of epitaxial GdN. J. Appl. Phys., 106(063910), 2009.

[18] F. Natali, B. J. Ruck, H. J. Trodahl, Do Le Binh, S. Vezian, B. Damilano, Y. Cordier, F. Semond, and C. Meyer. Role of magnetic polarons in ferromagnetic GdN. Phys. Rev. B, 87(035202), 2013.

[19] N. O. V. Plank, F. Natali, J. Galipaud, J. H. Richter, M. Simpson, H. J. Trodahl, and B. J. Ruck. Enhanced Curie temperature in N-deficient GdN. Appl. Phys. Lett., 98(112503), 2011.

[20] R. Vidyasagar, T. Kita, T. Sakurai, and H. Ohta. Giant optical splitting in the spin-states assisting a sharp magnetic switching in GdN thin films. Appl. Phys. Lett., 102(222408), 2013. 
[21] A. Punya, T. Cheiwchanchamnangij, A. Thiess, and W. R. L. Lambrecht. First-principles study of nitrogen vacancies in GdN. MRS Proc., 1290(DOI: http://dx.doi.org/10.1557/opl.2011.383), 2011.

[22] F. Natali, B. Ludbrook, J. Galipaud, N. Plank, S. Granville, A. Preston, B. L. Do, J. Richter, I. Farrell, R. Reeves, S. Durbin, J. Trodahl, and B. Ruck. Epitaxial growth and properties of GdN, EuN and SmN thin films. Phys. Status Solidi C, 9(605), 2012.

[23] E.-M. Anton, B. J. Ruck, C. Meyer, F. Natali, H. Warring, F. Wilhelm, A. Rogalev, V. N. Antonov, and H. J. Trodahl. Spin/orbit moment imbalance in the near-zero moment ferromagnetic semiconductor SmN. Phys. Rev. B, 87(134414), 2012.

[24] J. H. Richter, B. J. Ruck, M. Simpson, F. Natali, N. O. V. Plank, M. Azeem, H. J. Trodahl, A. R. H. Preston, B. Chen, J. McNulty, K. E. Smith, A. Tadich, B. Cowie, A. Svane, M. van Schilfgaarde, and W. R. L. Lambrecht. Electronic structure of EuN: Growth, spectroscopy, and theory. Phys. Rev. B, 84(235120), 2011.

[25] B. J. Ruck, H. J. Trodahl, J. H. Richter, J. C. Cezar, F. Wilhelm, A. Rogalev, V. N. Antonov, Binh Do Le, and C. Meyer. Magnetic state of EuN: X-ray magnetic circular dichroism at the Eu $\mathrm{M}_{4,5}$ and $\mathrm{L}_{2,3}$ absorption edges. Phys. Rev. B, 83(174404), 2011.

[26] F. Natali, S. Vezian, S. Granville, B. Damilano, H.J. Trodahl, E.-M. Anton, H. Warring, F. Semond, Y. Cordier, S. V. Chong, and B. J. Ruck. Molecular beam epitaxy of ferromagnetic epitaxial GdN thin films. J. Cryst. Growth, 404(146), 2014.

[27] H. Warring, B. J. Ruck, H. J. Trodahl, and F. Natali. Electric field and photo-excited control of the carrier concentration in GdN. Appl. Phys. Lett., 102(132409), 2013.

[28] B. J. Ruck, F. Natali, N. O. V. Plank, Binh Do Le, M. Azeem, Maha Alfheid, C. Meyer, and H. J. Trodahl. The influence of nitrogen vacancies on the magnetic behaviour of rare-earth nitrides. Physica B: Condensed Matter, 407(2954), 2012.

[29] J. W. Orton and C. T. Foxon. The electron mobility and compensation in n-type GaN. Semicond. Sci. Technol., 13(310), 1998.

[30] C.-M. Lee, H. Warring, S. Vezian, B. Damilano, S. Granville, M. Al Khalfioui, Y. Cordier, H. J. Trodahl, B. J. Ruck, and F. Natali. 
Highly resistive epitaxial Mg-doped GdN thin films. Appl. Phys. Lett., 106(022401), 2015.

[31] A. Zehe, A. Ramirez, and B. W. Muller. Thermal response of a Knudsen-type effusion source to sudden heating-power changes. Superficies y vacio, 13(72), 2001.

[32] N. F. Ramsey. Molecular Beams. Oxford University Press, Oxford, 1956.

[33] W.K. Liu and M.B. Santos (eds.). Thin Films: Heteroepitaxial Systems. World Scientific Publishing Company, Singapore, 1999.

[34] B. Heying, X. H. Wu, S. Keller, Y. Li, D. Kapolnek, B. P. Keller, S. P. DenBaars, and J. S. Speck. Role of threading dislocation structure on the x-ray diffraction peak widths in epitaxial GaN films. Appl. Phys. Lett., 68(643), 1996.

[35] A. Dussaigne, B. Damilano, J. Brault, J. Massies, E. Feltin, and N. Grandjean. High doping level in Mg-doped GaN layers grown at low temperature. Appl. Phys. Lett., 103(013110), 2008.

[36] S. Khromov, D. Gregorius, R. Schiller, M. Wahl, M. Kopnarski, H. Amano, B. Monemar, L. Hultman, and G. Pozina. Atom probe tomography study of Mg-doped GaN layers. Nanotechnology, 25(275701), 2014.

[37] D. K. Schroder. Semiconductor Material and Device Characterization. John Wiley and Sons, New York, 1998.

[38] S. Blundell. Magnetism in Condensed Matter. Oxford University Press, New York, 2001.

[39] R. Vidyasagar, T. Kita, T. Sakurai, and H. Ohta. Electronic transitions in GdN band structure. J. Appl. Phys., 115(203717), 2014.

[40] J. M. D. Coey, M. Venkatesan, and C. B. Fitzgerald. Donor impurity band exchange in dilute ferromagnetic oxides. Nat. Mater., 4(173), 2005 .

[41] M. Schlipf, M. Betzinger, C. Friedrich, M. Lezaic, , and S. Blugel. HSE hybrid functional within the FLAPW method and its application to GdN. Phys. Rev. B, 84(125142), 2011. 
[42] C. Friedrich, M. Betzinger, M. Schlipf, S. Blugel, and A. Schindlmayr. Hybrid functionals and GW approximation in the FLAPW method. $J$. Phys.: Condens. Matter, 24(293201), 2012.

[43] H. J. Trodahl, A. R. H. Preston, J. Zhong, B. J. Ruck, N. M. Strickland, C. Mitra, and W. R. L. Lambrecht. Ferromagnetic redshift of the optical gap in GdN. Phys. Rev. B, 76(085211), 2007.

[44] M. Marques, L. K. Teles, L. M. R. Scolfaro, J. R. Leite, J. Furthmuller, and $\mathrm{F}$. Bechstedt. Lattice parameter and energy band gap of cubic $\mathrm{Al}_{x} \mathrm{Ga}_{y} \mathrm{In}_{1-x-y} \mathrm{~N}$ quaternary alloys. Appl. Phys. Lett., 83(890), 2003.

[45] J. I. Pankove (ed.). Semiconductors and Semimetals Vol. 21 Part B. Academic Press, Orlando, Florida, 1984.

[46] A. Koo, F. Budde, B. J. Ruck, H. J. Trodahl, A. Bittar, A. Preston, and A. Zeinert. Photoconductivity in nanocrystalline GaN and amorphous GaON. J. Appl. Phys., 99(034312), 2006.

[47] S. J. Chung, Y. S. Lee M. Senthil Kumar, E.-K. Suh, and M. H. An. Characteristics of Mg-doped and In-Mg co-doped p-type GaN epitaxial layers grown by metal organic chemical vapour deposition. J. Phys. D: Appl. Phys., 43(185101), 2010.

[48] J. Z. Li, J. Y. Lin, H. X. Jiang, A. Salvador, A. Botchkarev, and H. Morkoc. Nature of Mg impurities in GaN. Appl. Phys. Lett., 69(1474), 1996.

[49] D. Seghier and H. P. Gislason. Correlation between deep levels and the persistent photoconductivity in Mg-doped GaN. J. Phys. D: Appl. Phys., 35(291), 2002.

[50] C. Johnson, J. Y. Lin, H. X. Jiang, M. Asif Khan, and C. J. Sun. Metastability and persistent photoconductivity in Mg-doped p-type GaN. Appl. Phys. Lett., 68(1808), 1996. 